\title{
Cortex
}

\section{A new ERP repetition paradigm to assess independence of regularity representations in the extrastriate cortex. --Manuscript Draft--}

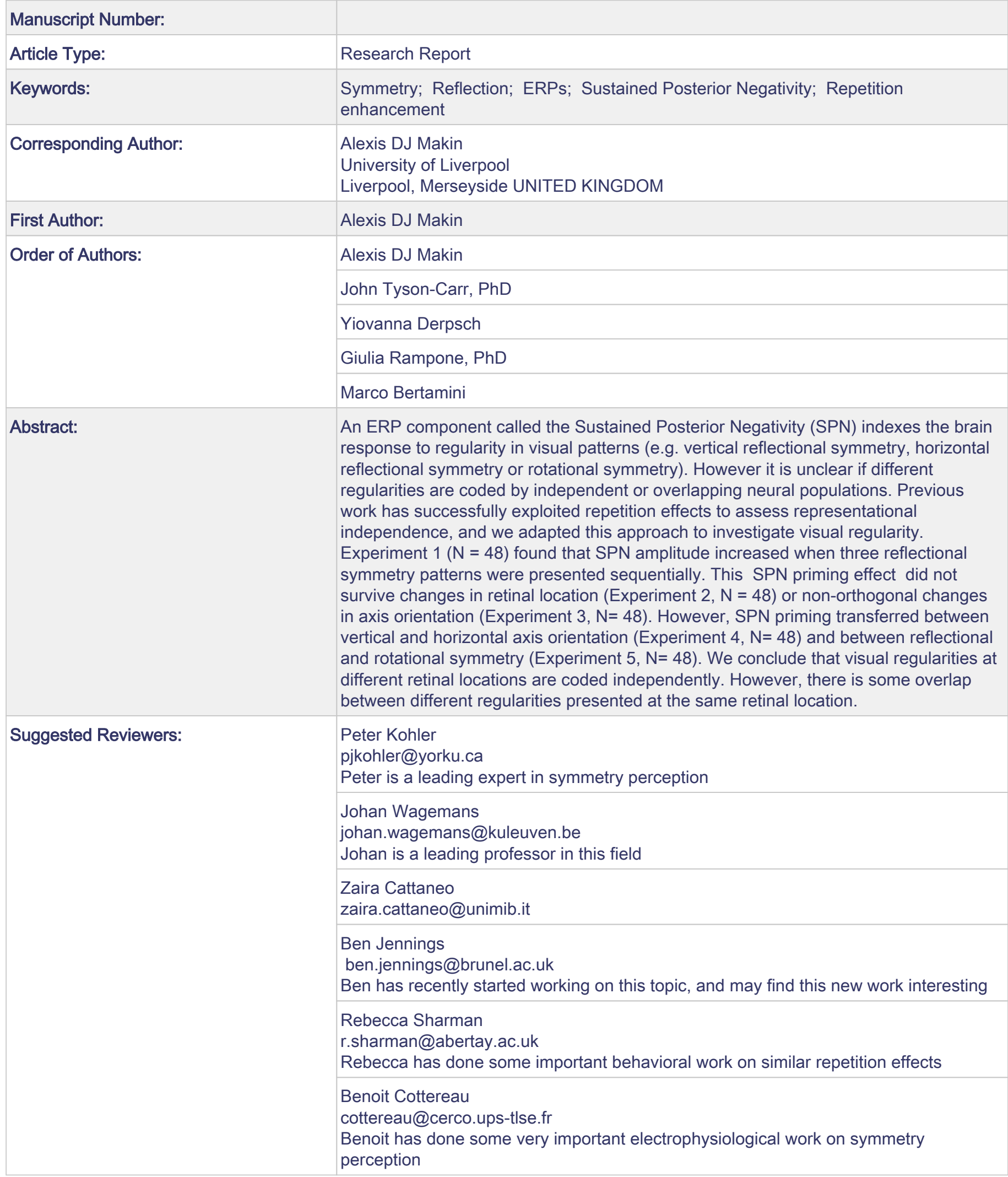


Dr Alexis D.J. Makin

Department of Experimental Psychology, University of Liverpool,

Liverpool,

United kingdom,

L72 7ZA

Email: alexis.makin@liverpool.ac.uk

Tel: 01517943909

19 March 2020

Dear Professor,

Please find our manuscript entitled: "A new ERP repetition paradigm to assess independence of regularity representations in the extrastriate cortex"

We have been examining the neural response to visual symmetry for 10 years. This work now has an international following, with several highly-cited publications (for review see, Bertamini, Silvanto, Norcia, Makin, \& Wagemans, 2018). This paper reports one of our most ambitious new projects, combining data from 240 EEG participants across 5 experiments. Using both established and cutting edge analysis, we extracted new insights into the processes of gestalt formation in the visual cortex.

We look forward to hearing from you,

Dr Alexis Makin

Bertamini, M., Silvanto, J., Norcia, A. M., Makin, A. D. J., \& Wagemans, J. (2018). The neural basis of visual symmetry and its role in mid- and high-level visual processing. Annals of the New York Academy of Sciences, 1426(1), 111-126. https://doi.org/10.1111/nyas.13667 
A new ERP repetition paradigm to assess independence of regularity representations in the extrastriate cortex.

Alexis D.J. Makin, John Tyson-Carr, Yiovanna Derpsch, Giulia Rampone and Marco Bertamini

1: University of Liverpool,

Department of Psychological Sciences,

Eleanor Rathbone Building,

University of Liverpool,

Liverpool,

L69 7ZA,

United Kingdom

Corresponding Author

Alexis D.J. Makin

Alexis.makin@liverpool.ac.uk 


\begin{abstract}
An ERP component called the Sustained Posterior Negativity (SPN) indexes the brain response to regularity in visual patterns (e.g. vertical reflectional symmetry, horizontal reflectional symmetry or rotational symmetry). However it is unclear if different regularities are coded by independent or overlapping neural populations. Previous work has successfully exploited repetition effects to assess representational independence, and we adapted this approach to investigate visual regularity. Experiment $1(N=48)$ found that SPN amplitude increased when three reflectional symmetry patterns were presented sequentially. This SPN priming effect did not survive changes in retinal location (Experiment 2, $N=48$ ) or non-orthogonal changes in axis orientation (Experiment $3, \mathrm{~N}=48$ ). However, SPN priming transferred between vertical and horizontal axis orientation (Experiment 4, $\mathrm{N}=48$ ) and between reflectional and rotational symmetry (Experiment $5, \mathrm{~N}=48$ ). We conclude that visual regularities at different retinal locations are coded independently. However, there is some overlap between different regularities presented at the same retinal location.
\end{abstract}

\title{
Keywords
}

Symmetry, Reflection, ERPs, Sustained Posterior Negativity, Repetition enhancement 


\section{Introduction}

Many animal species show a preference for visual symmetry (Møller, 1992; Wignall, Heiling, Cheng, \& Herberstein, 2006), possibly because it is useful for object recognition (Machilsen, Pauwels, \& Wagemans, 2009) and/or healthy mate selection (Grammer, Fink, Møller, \& Thornhill, 2003; Rhodes, Proffitt, Grady, \& Sumich, 1998). Reflectional symmetry is just one type of visual regularity produced by a rigid transformation: Mach (1886) noted that other rigid transformations, such as rotations and translations are equally regular in a mathematical sense, but are less obvious to human observers. Psychophysical work has confirmed the perceptual advantage for reflection, especially when the axis is vertical (Barlow \& Reeves, 1979; Treder, 2010; Wagemans, 1995). There are now several plausible models showing how reflection can be extracted from arrays of spatial frequency and orientation tuned filters (Dakin \& Hess, 1997; Mancini, Sally, \& Gurnsey, 2005; Poirier \& Wilson, 2010; Rainville \& Kingdom, 2000).

The neural response to visual regularity has also been studied extensively (for reviews see Bertamini \& Makin, 2014; Bertamini, Silvanto, Norcia, Makin, \& Wagemans, 2018; Cattaneo, 2017). EEG studies have consistently reported an ERP called the Sustained Posterior Negativity (SPN): amplitude is more negative at posterior electrodes for symmetrical than asymmetrical stimuli (Höfel \& Jacobsen, 2007; Jacobsen \& Höfel, 2003; Makin, Wilton, Pecchinenda, \& Bertamini, 2012; Martinovic, Jennings, Makin, Bertamini, \& Angelescu, 2018). The SPN is found whether participants are actively discriminating regularity or when they perform other tasks (Makin, Rampone, \& Bertamini, 2015; Makin, Rampone, Morris, \& Bertamini, 2020; Makin, Rampone, Wright, Martinovic, \& Bertamini, 2014). In line with Mach's early observations, Makin, Pecchinenda, Rampone and Bertamini (2013) found that SPN amplitude was larger for reflectional than rotational symmetry. Subsequently Palumbo, 
Bertamini and Makin (2015) found a linear increase in SPN amplitude with the proportion of symmetry in symmetry + noise displays, and Makin et al. (2016) reported that SPN amplitude scales with perceptual goodness across a range of regularity types, including Glass patterns, translation and multiple reflections (van der Helm \& Leeuwenberg, 1996).

Source localization analysis suggests that the SPN is generated by the extrastriate visual cortex (Kohler, Clarke, Yakovleva, Liu, \& Norcia, 2016; Makin et al., 2012). This is consistent with $\mathrm{fMRI}$ work, which has identified a parametric response to symmetry in extrastriate regions V3, V3a, V4, VO1 and LOC (Bauer et al., 2015; Chen, Kao, \& Tyler, 2007; Keefe et al., 2018; Kohler et al., 2016; Kohler, Cottereau, \& Norcia, 2018; Sasaki, Vanduffel, Knutsen, Tyler, \& Tootell, 2005; Tyler et al., 2005; Van Meel, Baeck, Gillebert, Wagemans, \& Op de Beeck, 2019). Moreover, TMS studies have confirmed that disruption of LOC impairs symmetry discrimination (Bona, Cattaneo, \& Silvanto, 2015; Cattaneo, Mattavelli, Papagno, Herbert, \& Silvanto, 2011), and neuropsychological examinations have shown that early ventral stream lesions covering LOC cause symmetry blindness along with many other kinds of object agnosia (Milner et al., 1991).

Despite substantial progress, the nature of the extrastriate symmetry code remains unclear. One outstanding question is the degree of representational independence in the extrastriate network. Are different types of symmetry coded independently? Are reflections with differently oriented axes coded independently? What about reflections at different retinal locations? Filter models indirectly imply that there should be substantial independence whenever different retinotopic receptive fields are involved.

Following a well-established research strategy, we reasoned that stimulus repetition effects could provide some insight into neural independence (Miller, Li, \& Desimone, 1991). If prior presentation of stimulus $A$ has no effect on the response to stimulus $B$, then $A$ and $B$ 
are coded independently. Conversely, if prior presentation of stimulus A alters the neural response to stimulus $B$, then there is some overlap between $A$ and $B$ in the brain. Thus transfer of repetition effects tells us about the functional boundaries within the visual system (Kourtzi \& Kanwisher, 2001). We therefore examined the independence of different regularity representations measuring the SPN within a novel repetition paradigm.

To anticipate the results: Experiment 1 found a repetition effect we termed the SPN priming: SPN amplitude increased with three repeated presentations of reflectional symmetry. Subsequent experiments then examined transfer of SPN priming across visual dimensions. Experiment 2 found that SPN priming did not transfer between retinal locations. Likewise, Experiment 3 found that SPN priming did not transfer across non-orthogonal changes in axis orientation. Conversely, Experiment 4 found transfer between horizontal and vertical orientations, and Experiment 5 found transfer between reflection and rotational symmetry. Together, these experiments suggest some common processing of regularities presented at the same retinal location.

\section{Experiment 1. Repetition effects with different and identical reflections}

To establish the basic parameters of any SPN repetition effects, Experiment 1 examined whether the SPN is subject to repetition enhancement (that is, priming) or suppression (that is, adaptation). Experiment 1 also tested how SPN repetition effects are modulated by low-level visual properties of the successive patterns. Having established these foundations, subsequent experiments exploited repetition effects to probe independence of different regularity representations.

Both repetition enhancement and suppression have been shown in previous work. For instance, evoked responses often decline with repeated presentation. This is a basic form of 
memory in sensory systems, and it is an essential piece of evidence for influential Bayesian brain (Knill \& Pouget, 2004) and hierarchical predictive coding models (Clark, 2013; Rao \& Ballard, 1999). Repetition suppression can be shown in specific brain regions with fMRI (Clark, 2013; Kim, Biederman, Lescroart, \& Hayworth, 2009; Kourtzi \& Kanwisher, 2001) and also with electrophysiology (Grill-Spector, Henson, \& Martin, 2006). There are also cases of repetition enhancement. For instance, Henson, Shallice, \& Dolan (2000) found enhancement when participants are repeatedly presented with faces or abstract stimuli. Cognitive factors, such as familiarity, cause the system to switch between repetition suppression and enhancement modes. Given this background, it was not clear whether the SPN would be subject to repetition suppression or enhancement.

Trial structure and conditions of Experiment 1 are shown in Figure 1. Each trial began with a $1500 \mathrm{~ms}$ baseline. This was followed by a sequence of three abstract patterns (500 ms each, separated by $200 \mathrm{~ms}$ gaps). Patterns were either random or regular with 8-fold reflectional symmetry. We used 8-fold reflectional symmetry to maximise the SPN amplitude (Makin et al., 2016). Participants performed an oddball detection task, and identified the minority of trials with a grey blank disk at the second sequence position (Figure 1A). We used this secondary task because we were interested in the dynamics of spontaneous regularity coding (rather than repetition effects on cognitive classifications).

If the symmetry response is subject to repetition suppression, SPN amplitude should decline from presentations $1>2>3$. This would be consistent with some perceptual adaptation studies, where prolonged exposure to a regular grid causes subsequent grids to look less regular (Morgan, Mareschal, Chubb, \& Solomon, 2012; Ouhnana, Bell, Solomon, \& Kingdom, 2013; Yamada, Kawabe, \& Miyazaki, 2013). Moreover, other research has observed repetition suppression in LOC, where symmetry representations are coded (Kim et al., 2009). 
Alternatively, if the symmetry response is subject to repetition enhancement, SPN amplitude should increase from presentations $1>2>3$. This alternative might be expected in light of previous psychophysical work (Sharman \& Gheorghiu, 2017; Wenderoth, 1994).

Experiment 1 included two manipulations to test the specificity of SPN repetition effects. First, we contrasted simple and complex patterns, which differed in spatial frequency content and other low-level visual features (see left and right columns of Figure 1B). This was a between-subjects factor, with 24 participants in simple and complex conditions. Second, we examined repetition effects with identical and different exemplars. If repetition effects are driven by changes in the neural response to edges and elements (e.g. in V1) they should be greater in the identical condition, as exemplars hit the same receptive fields on each repeat. However, if repetition effects are driven by changes to the gestalt (e.g. in the LOC), they should be comparable in both conditions, or stronger in the different reflections condition, where new structure appears around pre-encoded axes.

It is likely that mere repetition of a stimulus, with three visual onsets and offsets, elicits some non-specific repetition response. Therefore we also included a RandRandRef condition, where a reflection pattern is presented third, following two randoms. The SPN response to this third reflection allows us estimate this non-specific repetition effect.

\section{Experiment 1 procedure}

General methods for all experiments are described at the end. Experiment 1 had 48 participants (age 18-43, mean age 20, 9 males, 4 left-handed). Each trial began with a 1500 ms blank baseline with white placeholder disk and red fixation dot in the centre. On most trials, three patterns were then shown for $500 \mathrm{~ms}$, separated by $200 \mathrm{~ms}$ intervals. On rare oddball trials, the second element of the sequence was a blank grey disk. On each trial, 
participants responded 'all patterns' or 'blank in the middle', using the $A$ and $L$ keys to enter their responses (Figure 1A). The meaning of the keys switched unpredictably, but there were an equal number of trials with each key mapping.

The experiment was divided in 15 blocks of 36 trials (540 trials in total). Participants had a break in between each block, and the electrodes were checked if necessary. Trials in each block were presented in random order. An additional single block was presented as practice (not included in EEG analysis). In the main experiment there were 240 different random trials, and 60 trials from each of the other conditions (Figure 1B). There were also 60 additional oddball trials (11.1\%) requiring a blank in the middle response. These oddball trials were not included in EEG analysis. The proportion of triplet types in each block was identical and thus matched the whole experiment. Participants gave the correct answer on $97 \%$ of trials. Trials were included in EEG analysis even if participants gave the wrong behavioural response. 
A

Time

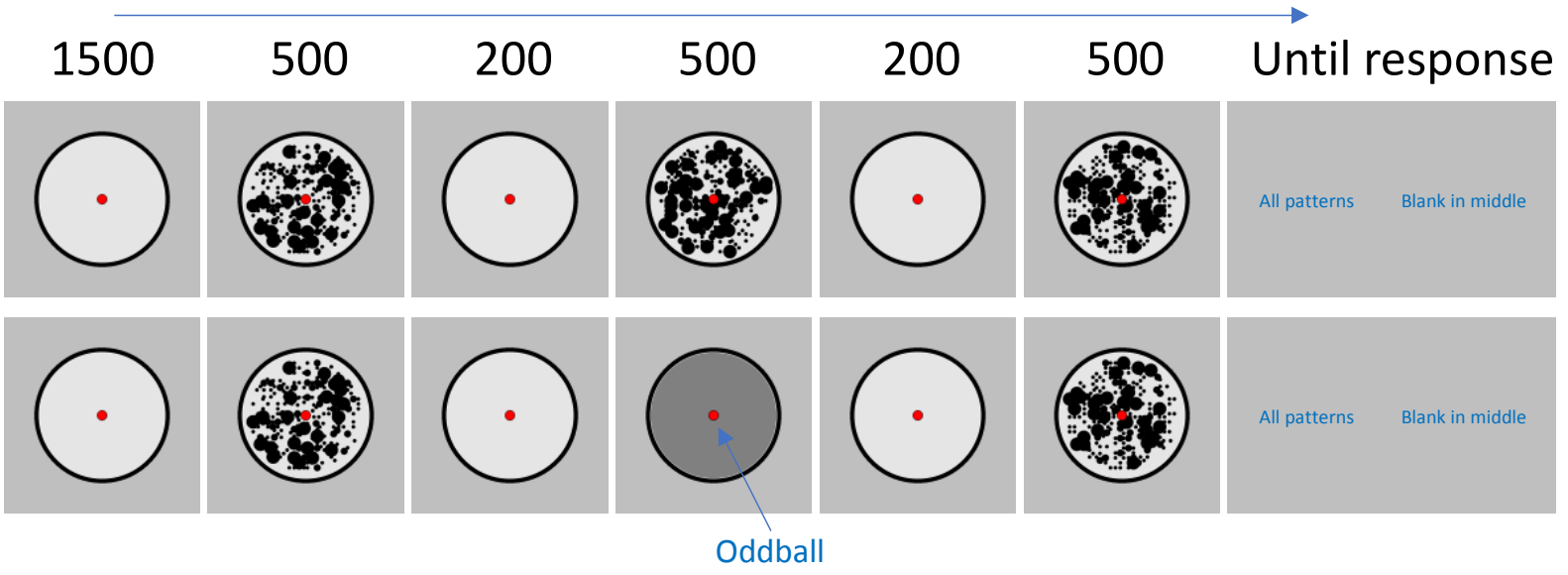

B Complex
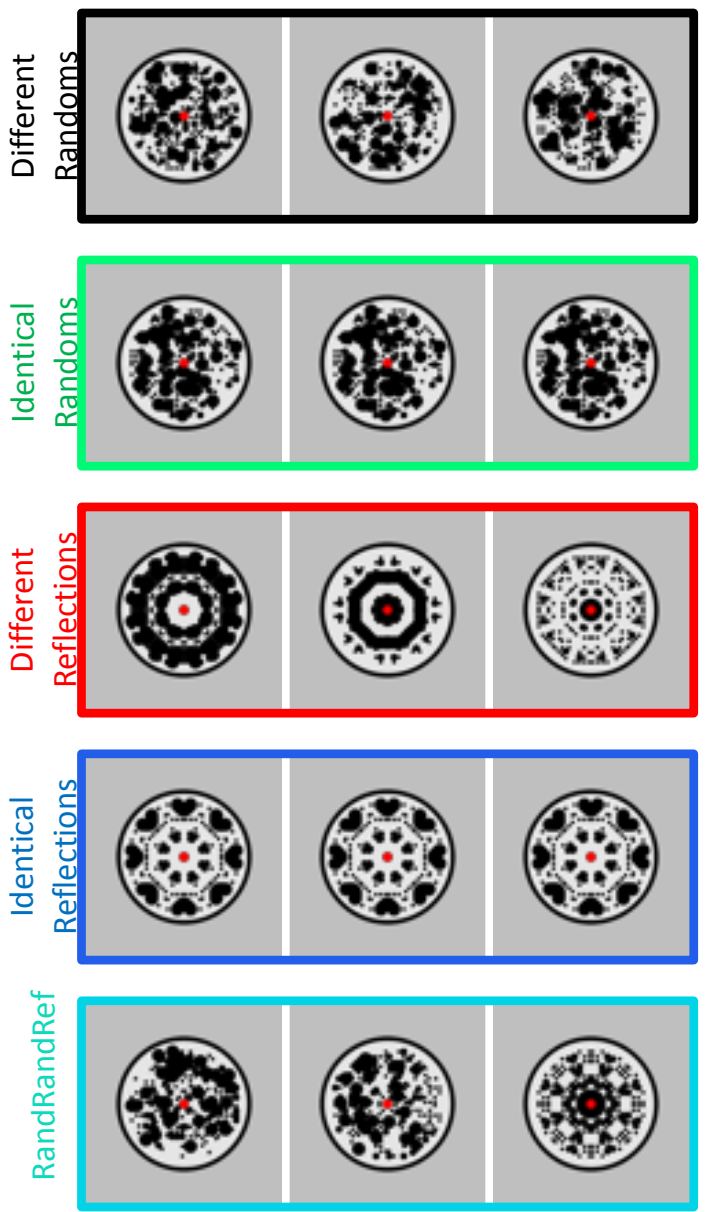

Simple
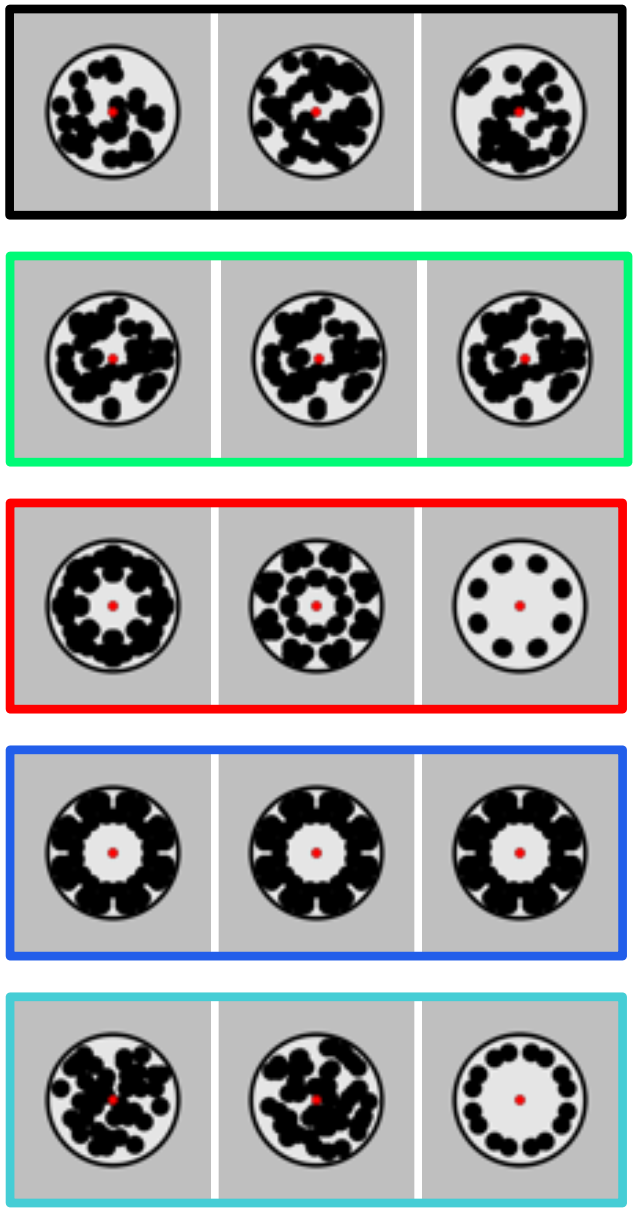

Figure 1. Experiment 1 method and stimuli. A) Trial structure in normal and oddball trials. All trials involved a sequence of three 500 ms presentations, separated by $200 \mathrm{~ms}$ gaps. Participants responded by pressing buttons for "All patterns" (all three presentations involved black and white patterns, as in the top row) or "Blank in the middle" (the second item in the sequence was blank, as in the lower row). B) Different triplets used. 24 participants were 
presented with complex patterns (left column) and another 24 were presented with simple patterns (right column). Individual patterns shown here are examples, in the real experiment each trial used different patterns. The coloured frames are matched with the colour of ERP waves in subsequent figures.

\section{Experiment 1 results}

Figure 2A shows grand-average ERP data from the posterior electrode cluster [PO7, PO3, PO4 and PO8], averaged over the between-subjects factor Pattern type (simple, complex). Unsurprisingly, there are three VEPs driven by the three sucessive pattern onsets. The SPN difference wave also had three peaks, although amplitude remained below zero throughout (Figure 2B). When three different reflections were presented, SPN amplitude increased with repetition (red trace). This repetition enhancement effect is referred to as SPN priming. When three identical patterns were presented, there was no SPN priming (blue trace). In the RandRandRef condition, the third pattern (a reflection) generated an SPN of intermediate amplitude (light blue trace).

To examine this selective SPN priming effect statistically, the difference from random wave was measured in the final $250 \mathrm{~ms}$ of each presentation interval and $100 \mathrm{~ms}$ into the ISI (First window $=250-600 \mathrm{~ms}$, Second window $=950$ to $1300 \mathrm{~ms}$, Third window $=1650$ to 2000 ms). Average SPN amplitude from these time windows is shown in Figure $2 \mathrm{C}$. Negative bars in Figure $2 \mathrm{C}$ represent mean amplitudes significantly lower than zero $(p<.001$, see $95 \%$ confidence intervals). 


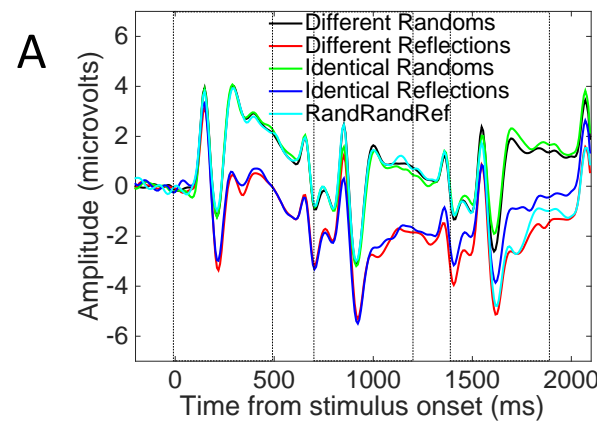

$\mathrm{B}$

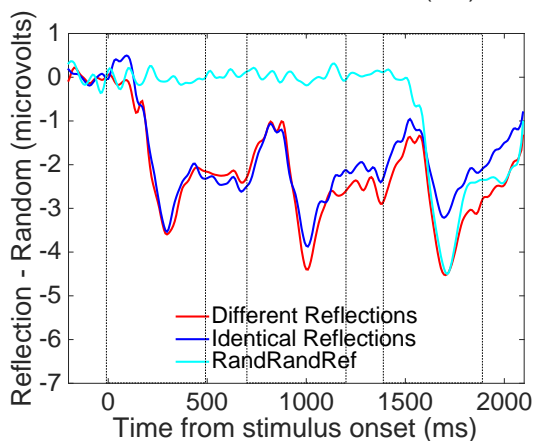

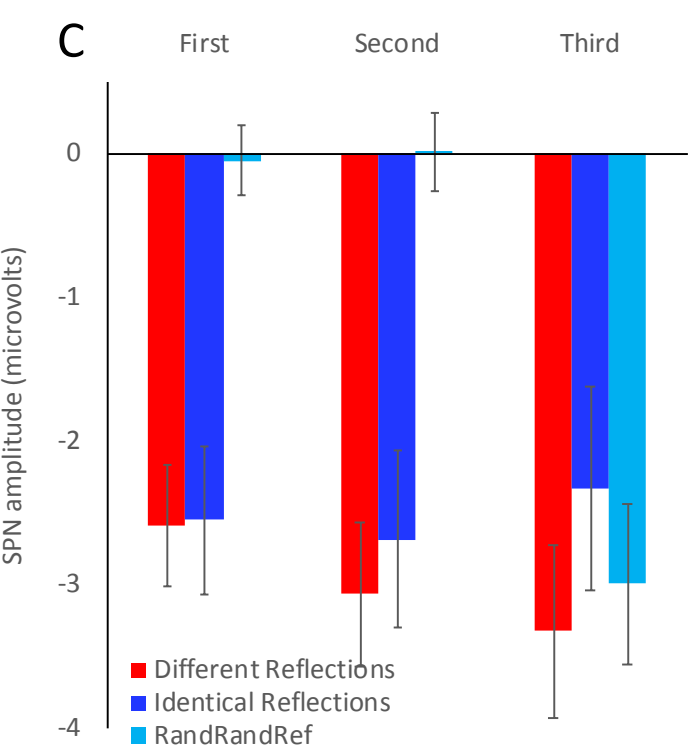

Figure 2. Experiment 1 results. A) Grand Average ERPs from electrode cluster $01, P O 7,02$ and PO8. Colour corresponds to coloured outlines in Figure 1. Results are averaged over the Complex and Simple patterns. B) The SPN shown as a difference wave. The intervals where the patterns were visible on the screen are shown with dotted boxes. C) Mean SPN for first, second and third intervals. Error bars $=95 \%$ confidence intervals (if they do not cross zero, the amplitude difference from the Randoms sequence is significant at the .05 level). Note SPN priming in the different reflections condition (red), but not in the identical reflections condition (dark blue).

Effects were analysed with a mixed ANOVA. There were two within-subjects factors [3 Sequence position (first, second, third) X 3 Sequence type (different reflections, identical reflections, RandRandRef)] and one between-subjects factor [2 Pattern complexity (complex, simple)]. There were main effects of Sequence position $\left(F(2,92)=56.179, p<.001, \eta_{p}{ }^{2}=.550\right)$ and Sequence type $\left(F(2,92)=37.906, p<.001, \eta_{p}^{2}=.450\right)$ and an interaction between Sequence position and Sequence type $\left(F(2.752,126.594)=54.052, p<.001, \eta_{p}{ }^{2}=.540\right)$. There was no effect of the between-subjects factor Complexity $(F(1,46)=3.627, p=.063)$ and no interactions involving Complexity $(p>.116)$.

We next conducted a two-factor repeated measures ANOVA on the different and identical reflection conditions [Sequence position (first, second, third) $X$ Sequence type 
(different reflections, identical reflections)]. There was a significant interaction ( $F(1.520$, $\left.71.437)=7.945, p=.002, \eta_{p}^{2}=.145\right)$. This was due to a significant linear effect of Sequence position in the different reflections condition $\left(F(1,47)=13.674, p=.001, \eta_{p}{ }^{2}=.225\right)$ but not in the identical reflections condition $(F<1)$.

Next, we analysed amplitude of the third SPN with one factor repeated measures ANOVA (Previous pattern (different reflections, identical reflections, randoms]. There was a significant influence of Previous pattern $\left(F(2,94)=4.570, p=.013, \eta_{p}{ }^{2}=.089\right)$. The SPN was larger after two different reflection exemplars than two identical reflection exemplars ( $\mathrm{t}(47)$ $=2.657, p=.011)$. The third pattern from the RandRandRef sequence (e.g. the reflection) generated an SPN of intermediate amplitude, which did not significantly differ from the other conditions (different reflections $\mathrm{t}(47)=-1.136, p=.262$; identical reflections sequence $\mathrm{t}(47)=$ $1.980, p=.054)$. This aspect of the results was somewhat inconclusive. However, it does highlight the fact that the SPN priming effect is not large: prior presentation of reflections did not dramatically increase the amplitude neural response compared to prior presentation of randoms.

This basic ERP analysis was based on a subset of electrodes and time windows. This is potentially problematic, so we also employed mass univariate and global field power (GFP) analysis, which incorporates all electrodes and time points (see supplementary materials 1). This suggested that the crucial results were not problematically dependent electrode and time window choice.

\section{Cortical sources of the SPN priming effect}

We interpreted the SPN priming effect as an increase in amplitude of the bilateral extrastriate symmetry response. However, an alternative explanation is that SPN priming is 
caused by additional activations emerging elsewhere in the brain towards the end of the trial. ERPs from different cortical sources summate at the scalp: another repetition sensitive ERP, generated elsewhere in the cortex, may be responsible for the observed priming effect at posterior electrodes. We thus examined whether the SPN priming effect happens within the extrastriate symmetry network with a source dipole analysis based on Classical LORETA recursively applied (CLARA) (Pascual-Marqui, Michel, \& Lehmann, 1994). The analysis used here was based on other recent work (Tyson-Carr et al., 2018)

A source dipole model comprising of two bilateral sources within the extrastriate regions explained $94.7 \%$ of variance. Both ECD1 (left Brodmann area 19; approximate Talairach coordinates $-\mathrm{x}=-26.9, \mathrm{y}=-75.9, \mathrm{z}=-12.9$ ) and ECD2 (right Brodmann area 19; approximate Talairach coordinates $-x=26.9, y=-75.9, z=-12.9)$ were located within the fusiform gyrus. Thus, it appeared that this extrastriate region was the only significant generator of symmetry-specific cortical activity. The final model is detailed in Figure 3A, and the resulting source waveforms for each ECD are illustrated in Figure 3B. In these source waveforms there was a selective SPN priming effect in the different reflections condition (replicating the sensor level analysis).

This was confirmed with a repeated measures ANOVA [Sequence position (first, second, third) X Sequence type (different reflections, identical reflections) X Hemisphere (left $(E C D 1)$, right $(E C D 2)]$. The apparent right lateralization in Figure $3 C$ was not significant ( $(1,47)=3.651, p=.062)$. However, there was a main effect of Sequence position $(F(1.584$, $\left.74.426)=5.375, p=.011, \eta_{p}{ }^{2}=.103\right)$, as well as a Sequence position $X$ Sequence type interaction $\left(F(1.712,80.443)=4.407, p=.020, \eta_{p}{ }^{2}=.086\right)$. There was an effect of Sequence position in the different reflections condition $\left(F(1.456,68.453)=11.731, p<.001, \eta_{p}{ }^{2}=.200\right)$, 
but not in the identical reflections condition $(F(1.731,81.355)=1.138, p=.319)$. There were no other effects or interactions $(p>0.211)$.

In sum, the selective priming effects originally observed in sensor level analysis were found within this pair of bilateral extrastriate sources. We thus conclude that the SPN priming effect happens within the extrastriate symmetry network, and does not reflect effects generated elsewhere in the brain.

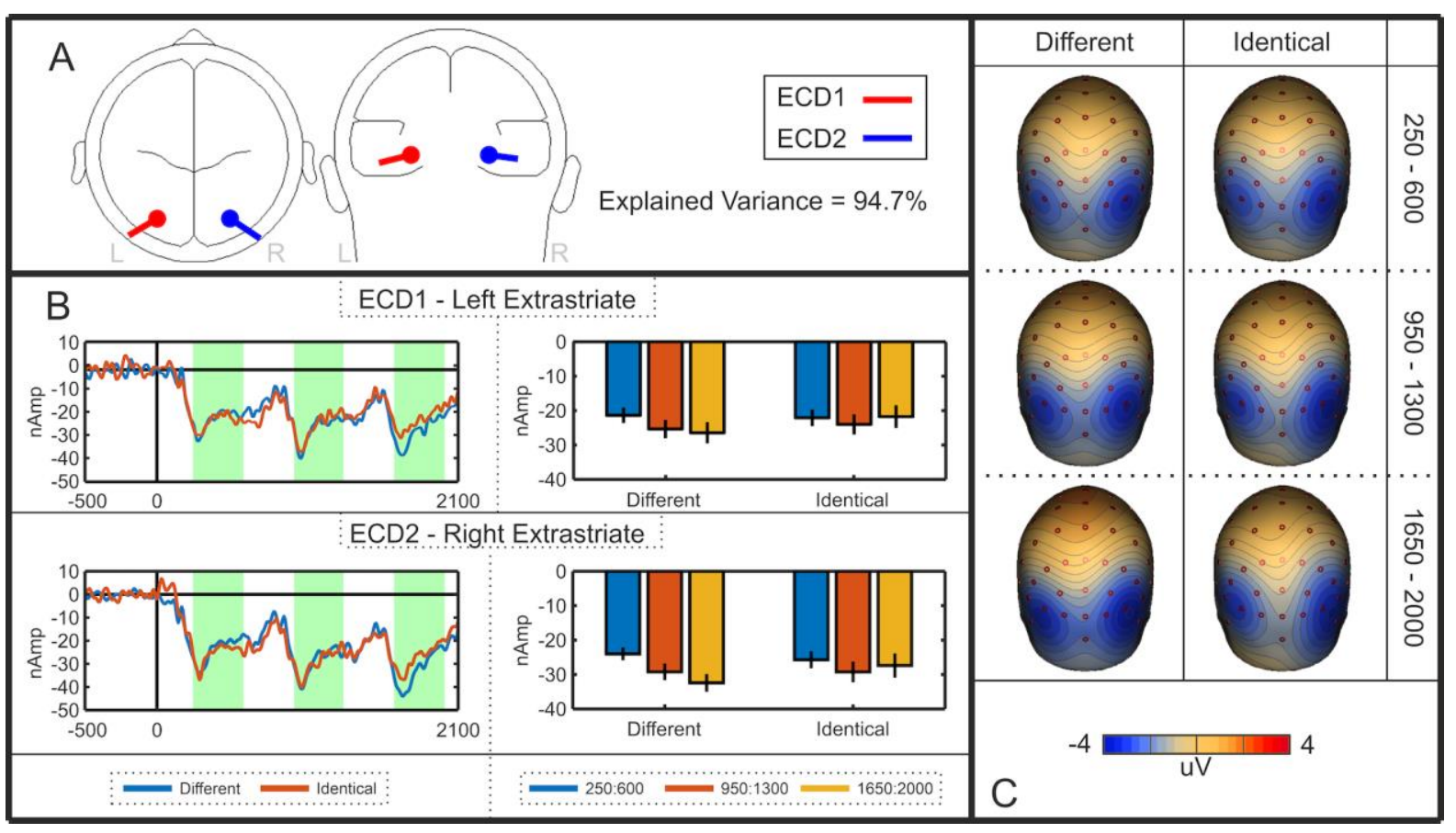

Figure 3. Experiment 1 source dipole model. A) Approximate locations of Equivalent Current Dipoles (ECDs) in the left and right extrastriate cortex. B) Left and right ECD source waveforms for the different and identical reflection sequences. The intervals used for statistical analysis are shown in green. Bar graphs indicate the mean activity in these intervals (error bars $=+/-1$ $S D$ ). Note the selective SPN priming effect in these extrastriate source waveforms. C) Rear view scalp difference maps (Different Reflection - Different Random; Identical Reflection - Identical Random). Each row shows topographies from first, second and third intervals.

\section{Experiment 1 Discussion}

Experiment 1 found an SPN priming effect for the sequence of three different reflections: SPN amplitude increased (i.e. became more negative) from presentations 1 to 3 . However, there 
was no SPN priming effect for the sequence of three identical reflections. This selectivity suggests that SPN priming is due to facilitation of the symmetry response, not facilitation of the response to individual elements. The results were similar for complex and simple patterns, despite the disparate image statistics. We thus conclude that the both SPN and SPN priming are unaffected by large variation of spatial frequency and other low-level visual features (an interesting result in its own right for SPN researchers). Finally, source-dipole analysis confirmed that SPN priming reflects increasing activation within the extrastriate symmetry network, rather than elsewhere in the brain.

The nature of SPN priming requires clarification: We did not observe three discrete SPNs. Instead, we observed a long difference wave, beginning 200 ms after the first pattern in the sequence. That is, although there was a stimulus-driven oscillation, it did not return to zero between each presentation. Nevertheless, we can say that the brain response to symmetry was selectively enhanced by repetition of novel exemplars, but not identical exemplars.

It seems that there is something special about the onset of new regularity of the same type, which 'tops up' the SPN generators. This is consistent with some behavioural findings. Sharman and Gheorghiu (2017) found that symmetry discrimination improved when elements moved to new positions or rapidly flickered on and off. This implies that symmetry detection benefits from new positional information around a pre-encoded axis. Niimi, Watanbe and Yokosawa (2008) also found a perceptual advantage for dynamic stimuli.

Another recent SPN study by Bertamini, Rampone, Oulton, Tatlidil and Makin (2019) found relevant effects. Two patterns were separated by a one second ISI. The SPN for pattern 2 was larger in the category repeat condition (different examples of reflectional symmetry) 
than in the exemplar repeat condition (identical patterns). This again shows that SPN priming only happens when novel exemplars are shown in succession.

\section{Experiment 2: Repeated and changing retinal locations}

Having established some fundamental parameters of the SPN priming effect in Experiment 1, Experiment 2 tested whether SPN priming transfers between left and right visual hemifields. We expected no inter-hemispheric transfer of SPN priming. Indeed, there is some experimental evidence to suggest that regularities presented in different retinal locations are functionally independent. For instance, Wright, Makin and Bertamini (2017) presented symmetrical patterns on the left or right of fixation, and recorded an SPN in the contralateral hemisphere only. This contralateral SPN was not altered by the pattern presented to the ipsilateral hemisphere (symmetry, asymmetry or nothing).

Experiment 2 used the same 8-fold reflections as Experiment 1. On each presentation in the triplet, a pair of patterns was presented with one pattern on either side of central fixation (therefore, a trial involved a triplet of pairs). Patterns were $5^{\circ}$ in diameter, and the gap between the left and right patterns was also $5^{\circ}$ (Figure 4). This ensured that symmetry information was presented in the opposite hemifield, well outside the putative foveal confluence. Participants fixated centrally, and fixation was monitored online with an eye tracker. We analysed eye data to confirm that participants did not routinely break central fixation or move their eyes towards the symmetrical patterns (see supplementary materials 2).

SPNs were computed as difference from the double random condition (black framed triplets in Figure 4). The crucial comparison was between repeated location conditions, where all three reflections were on one side (either the left or right, red framed triplets in Figure 4), 
and changing location conditions, where reflection switched from left to right or vice versa (green framed triplets in Figure 4). Note that the total amount of reflection presented in a trial was identical in repeated and changing location conditions. Therefore, if the extrastriate network codes regularity independently of retinal location, the SPN priming effect would be the same in both conditions.

\section{Experiment 2 procedure}

Another 48 participants were involved in Experiment 2 (age 16-43, mean age 22, 11 male, 8 left-handed). We tested 24 participants in the simple pattern condition and 24 in the complex pattern conditions. There were 30 blocks of 14 trials (120 random trials, and 60 of each of the other 4 conditions). There were 60 additional oddball trials (14.285\%) which were not included in EEG analysis. Participants performed the same oddball discrimination task as in Experiment 1, although blank oddballs now involved presentation of two blank disks. Participants gave the correct answer in $96 \%$ of trials. 

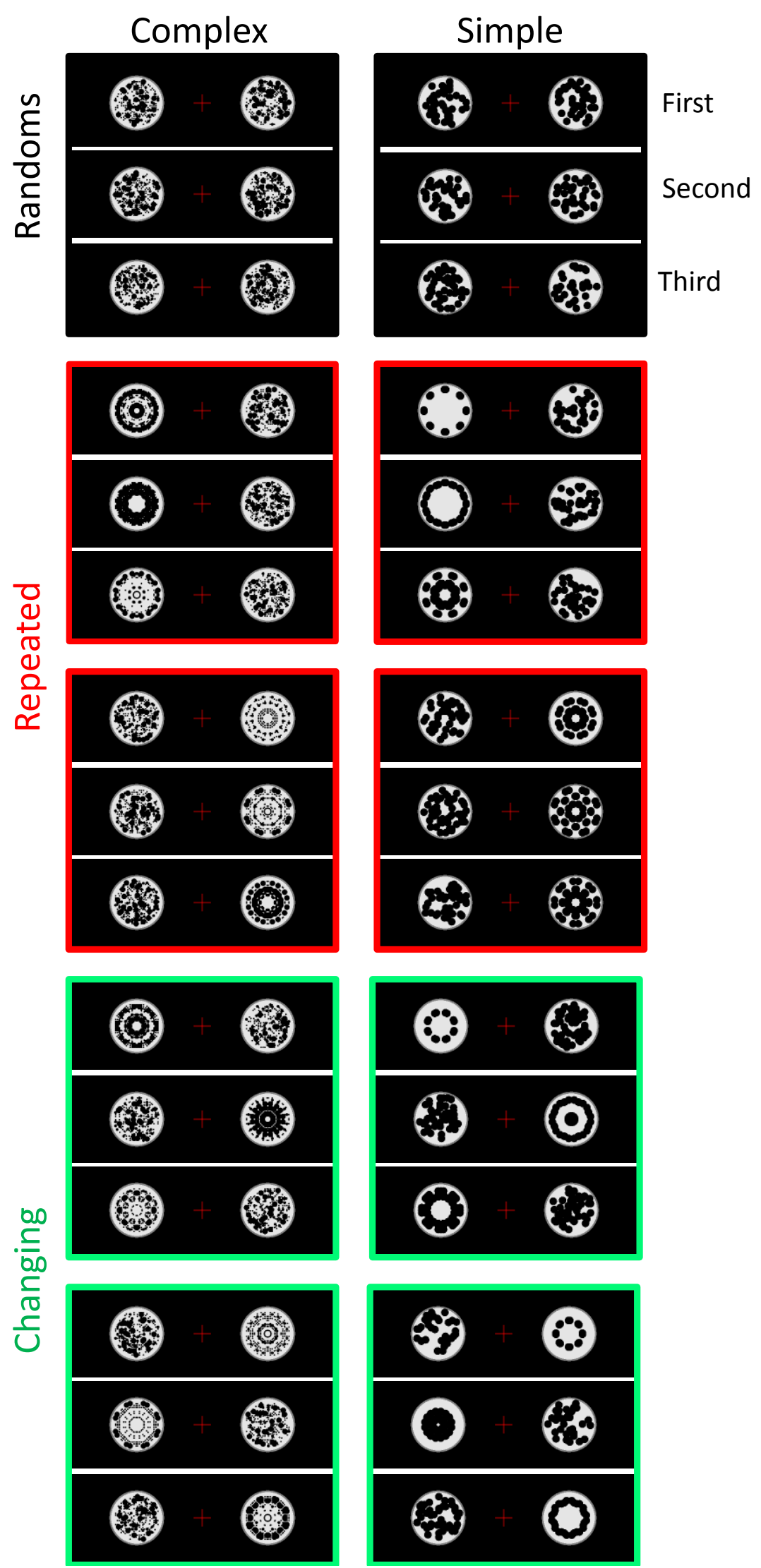

Figure 4. Experiment 2 stimuli. Each triplet had three pairs, with one pattern on the left of fixation, and one on the right of fixation. 24 participants were presented with complex pattern pairs (left column) and another 24 were presented with simple pattern pairs (right column). 


\section{Experiment 2 results.}

Results from repeated and changing location conditions are shown in Figure 5. Electrodes were chosen to capture the contralateral SPN (P3, P5, PO3 and PO7 on the left, P4, P6, PO4 and PO8 on the right). The difference waves in Figure $5 \mathrm{~B}$ and $6 \mathrm{C}$ represent the difference from the double random condition (where both left and right patterns were random). There was a clear SPN priming effect when the location of reflections was repeated, but not when location of reflections changed between visual hemifields.

A mixed ANOVA confirmed these impressions. There were two within-subjects factors [Consistency (repeated locations, changing locations) X Sequence position (first, second, third)] and one between-subjects factor [2 (Pattern complexity (complex, simple)]. This found main effects on Consistency $\left(F(1,46)=8.027, p=.007, \eta_{p}{ }^{2}=.149\right)$ and a Sequence position $X$ Consistency interaction $\left(F(1.520,69.923)=4.259, p=.027, \eta_{p}{ }^{2}=.085\right)$. There was a significant linear effect of Sequence position in the repeated locations condition $(F(1,47)=6.345, p=$ $\left..015, \eta_{\mathrm{p}}^{2}=.119\right)$, but not in the changing locations condition $(F<1)$. There were no other effects or interactions $(p>.078)$.

Unfortunately signal-to-noise ratio in Experiment 2 was not sufficient for source dipole analysis, however the topographies in Figure 6 confirm that there was a contralateral SPN, always opposite the reflectional symmetry. The selective contralateral SPN priming effect in the repeated locations condition can be seen in the left columns of Figure 6, and mass univariate analysis replicated the selective SPN priming (supplementary materials 1). 


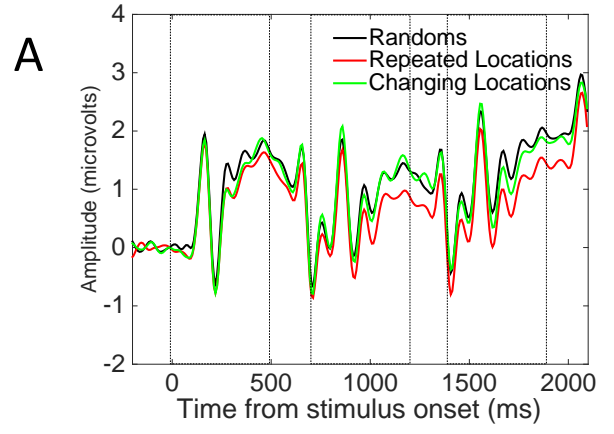

B

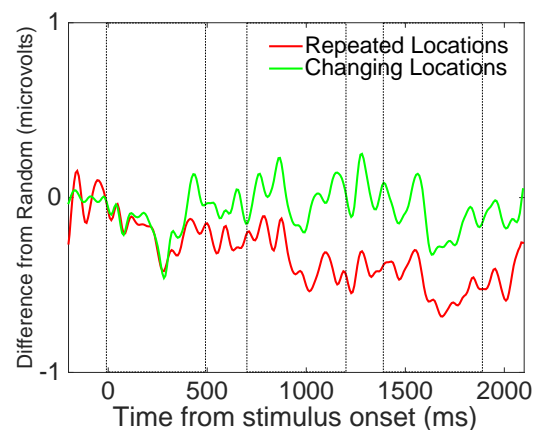

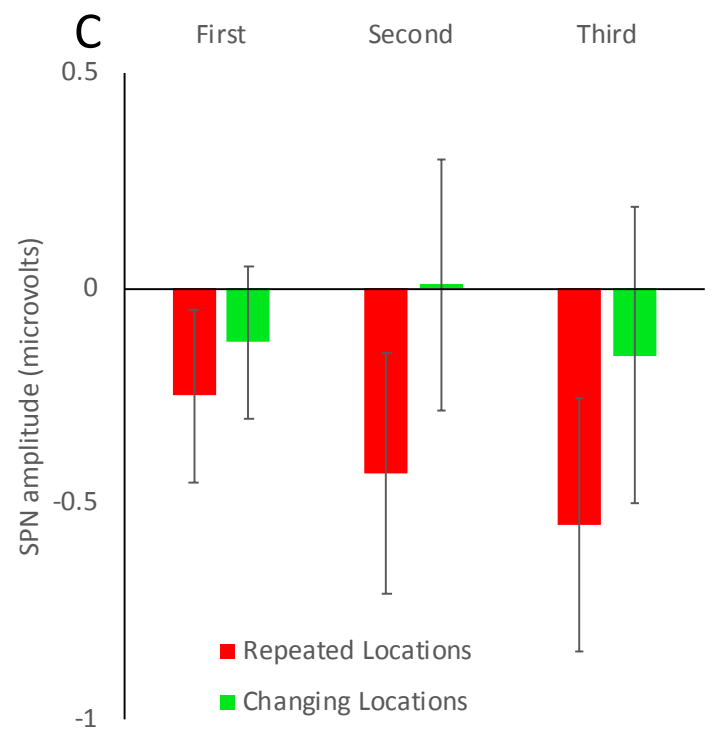

Figure 5. Experiment 2 results. Conventions are the same as Figure 2. A) ERPs from posterior electrode clusters (P3, P5, PO3 and PO7 on the left and P4, P6, PO4 and PO8 on the right). $B$ ) Difference waves as difference from the double random condition. C) Mean SPN for first, second and third intervals. Error bars $=95 \%$ confidence intervals. Note SPN priming in the repeated locations condition (red), but not in the changing locations condition (green).

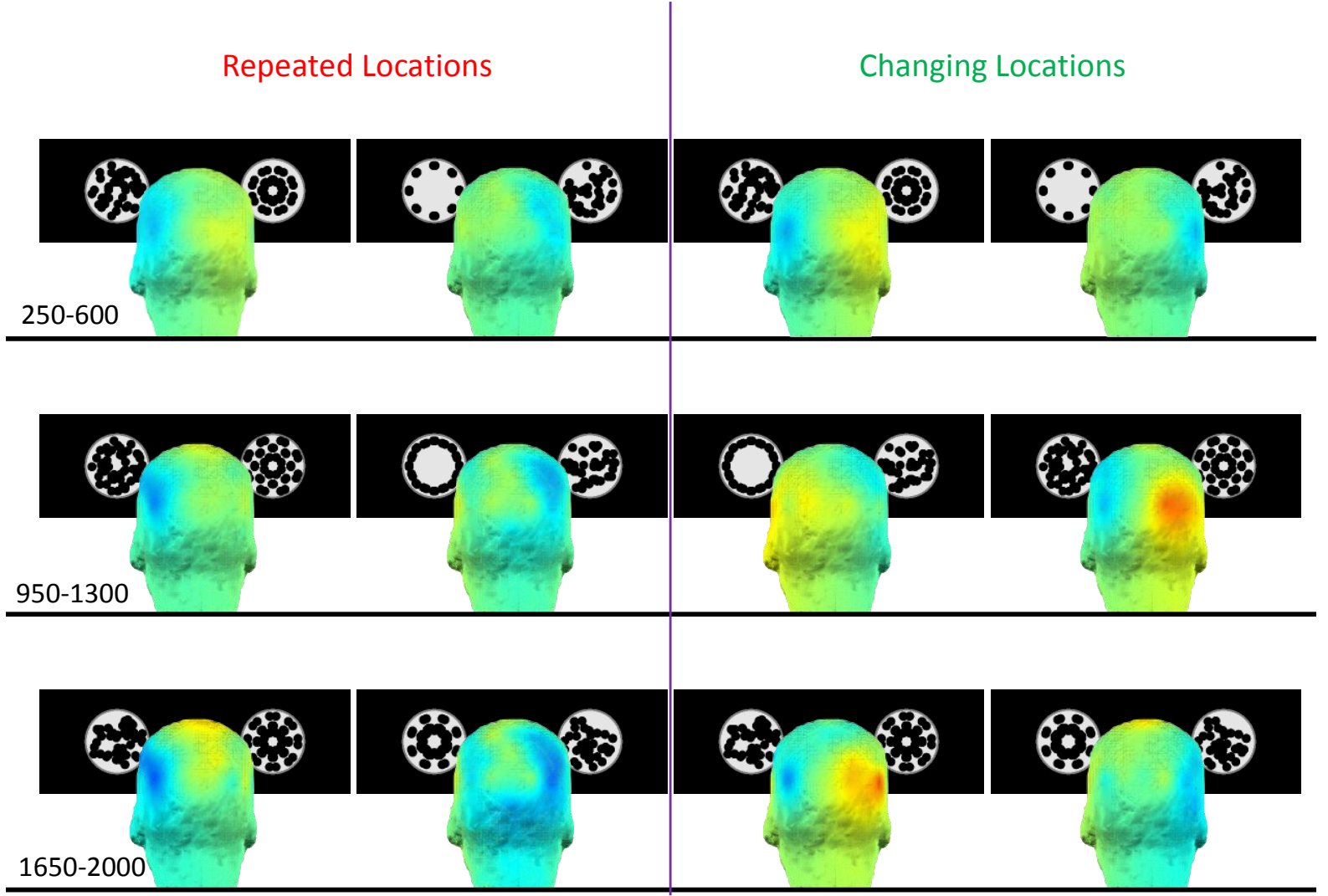


Figure 6. Rear-view topographic maps from Experiment 2, depicted as if the head is looking at the screen in front (not to scale) with simple pattern examples. Each row shows topographies from first, second and third intervals. Note the contralateral SPNs on the opposite side to reflection, and the increase in contralateral SPN amplitude across intervals in the repeated location conditions only (left columns).

\section{Experiment 2 Discussion}

Experiment 2 replicated the contralateral SPN from Wright et al. (2017), albeit at slightly more circumscribed clusters of bilateral posterior electrodes. As predicted, there was an SPN priming effect when reflectional symmetry was repeated in the same location, but not when it switched between left and right visual hemifields. This suggests that there are independent symmetry sensitive networks in each cerebral hemisphere and that cross talk between the hemispheres is minimal.

\section{Experiment 3. Repeated and changing orientations}

If the visual symmetry code is tightly linked to retinotopic maps (as suggested by Experiment 2), we should find SPN priming effect when axis orientation is repeated on each presentation, but not when it varies. This was tested in Experiment 3. The task was the same as Experiment 1 and 2. All stimuli were presented centrally at fixation. This experiment required one-fold reflection (Figure 7). On the first presentation of the triplet, axis orientation angle was set randomly between 0 (vertical) and 90 (horizontal). In the repeated orientation sequences, the chosen orientation was repeated on the second and third presentations. In the changing orientation sequence, orientation was set randomly again on the second and third presentation (with the constraint that subsequent orientations had to be separated by more than 10 degrees). 


\section{Experiment 3 procedure}

Experiment 3 involved another group of 48 participants (age 18-51, mean age 22, 9 male, 5 left-handed). There were 24 participants in the simple pattern condition and 24 in the complex pattern conditions. There were 18 trials in 15 blocks (120 random trials, 60 repeated and 60 changing orientation trials). There were 30 additional oddball trials (11.1\%). On average, participants gave the correct on $96 \%$ of the trials.

On consistent orientation trials, the orientation of the first pattern was set at random between 0 (vertical) and 90 degrees (horizontal), then the second and third patterns had the same orientation as the first. On changing orientation and random trials, the orientation of the second and third patterns was again set randomly between 0 and 90 degrees. However, there was a constraint that the absolute angular offset between one pattern and the next had to exceed 10 degrees. This prevented subsequent patterns in the changing sequence from having similar orientations by chance.

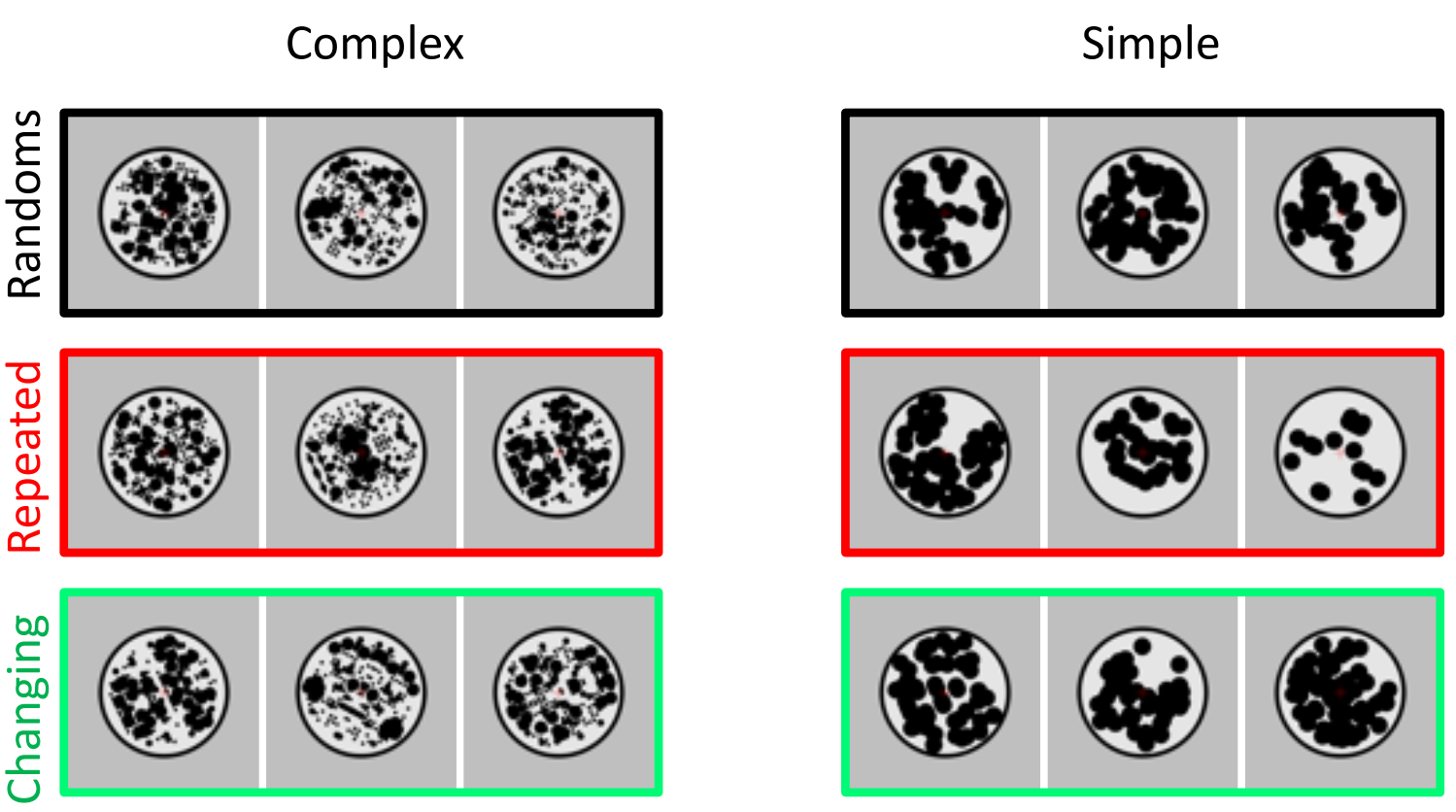

Figure 7. Experiment 3 stimuli. 24 participants were presented with complex patterns (left column) and another 24 were presented with simple patterns (right column). 


\section{Experiment 3 Results}

Grand average ERPs from Experiment 3 are shown in Figure 8. All six reflections generated a significant SPN $(p<.005)$. A mixed ANOVA revealed no significant main effects $(p>.060)$ and the expected Sequence position $X$ Sequence type interaction was marginal $(F(1.567,72.084)$ $=2.710, p=.086)$. However, there was an expected linear effect of Sequence position in the repeated orientation condition $\left(F(1,47)=5.838, p=.020, \eta_{\mathrm{p}}{ }^{2}=.110\right)$ but not in the changing orientations condition $(F<1)$. This suggests that SPN priming does not transfer between orientations that vary unpredictably. Furthermore, mass univariate analysis (supplementary materials 1) showed that SPN priming was specific to the repeated orientation condition. Again, the signal to noise ratio was not sufficient for source dipole analysis in Experiment 3.
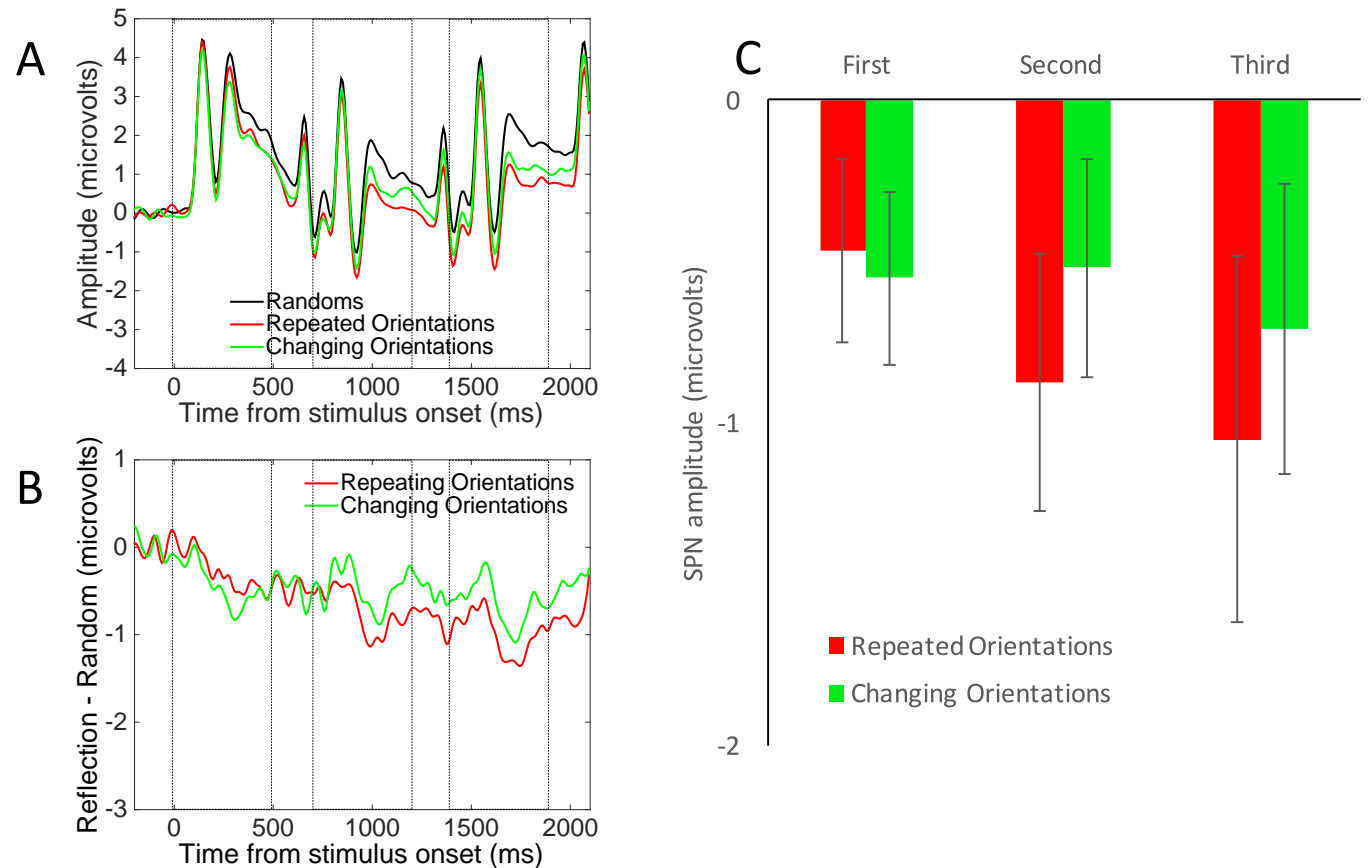

- Repeated Orientations

- Changing Orientations

Figure 8. Experiment 3 results. Note SPN priming in the repeated orientation conditions (Red) but not in the changing orientations condition (green). 


\section{Experiment 3 Discussion}

Results of Experiment 3 were straightforward. SPN priming was present in the repeated orientations condition, by not in the changing orientations condition. However, we are cautious about this difference, because there was no significant interaction (see Nieuwenhuis, Forstmann, \& Wagenmakers, 2011 for discussion of absent interactions in cognitive neuroscience). On the other hand, the selectivity in Experiment 3 was replicated with mass univariate analysis (see supplementary materials). Furthermore, the results are broadly consistent with those of Wenderoth (1994), who found that axis orientation priming enhanced symmetry detection in 2D dot patterns, and with Yamauchi et al. (2006), who found related results for 3D objects. It seems that the reflection code is not completely orientation invariant, and reflections with different orientations are extracted by different visual mechanisms.

\section{Experiment 4: Vertical and horizontal orientations}

Experiment 4 was closely related to Experiment 3, but all reflections were either vertical or horizontal. The repeated orientations condition either involved 3 verticals or 3 horizontals. The changing orientations condition involved switches between horizontal and vertical or vice versa (Figure 9).

Previous behavioural research shows that orthogonal axes might be more perceptually coupled than non-orthogonal axes. Treder, van der Vloed and van der Helm (2011) examined symmetry discrimination when patterns were preceded by same axis primes, orthogonal axis primes or non-orthogonal axis primes. They found a similar facilitation for same and orthogonal axis primes, but negative priming for non-orthogonal 
primes. Treder et al.'s result suggests that SPN priming should transfer between orthogonal horizontal and vertical reflections in Experiment 4.

\section{Experiment 4 procedure}

Another 48 participants were recruited (age 18-62, mean age 23, 8 male, 2 lefthanded). Experiment 4 had the same design as Experiment 3. There were 24 participants in the simple pattern condition and 24 in the complex pattern conditions. The consistent orientation conditions involved sequences of either three verticals (30 repeats) or three horizontals (30 repeats) (these trials were averaged in EEG analysis). The changing orientation conditions involved sequences of Vertical Horizontal Vertical (30 repeats), or Horizontal, Vertical Horizontal (30 repeats) (these were also averaged in EEG analysis). There were 30 additional oddball trials. On average participants gave the correct answer in $98 \%$ of trials. 
Complex
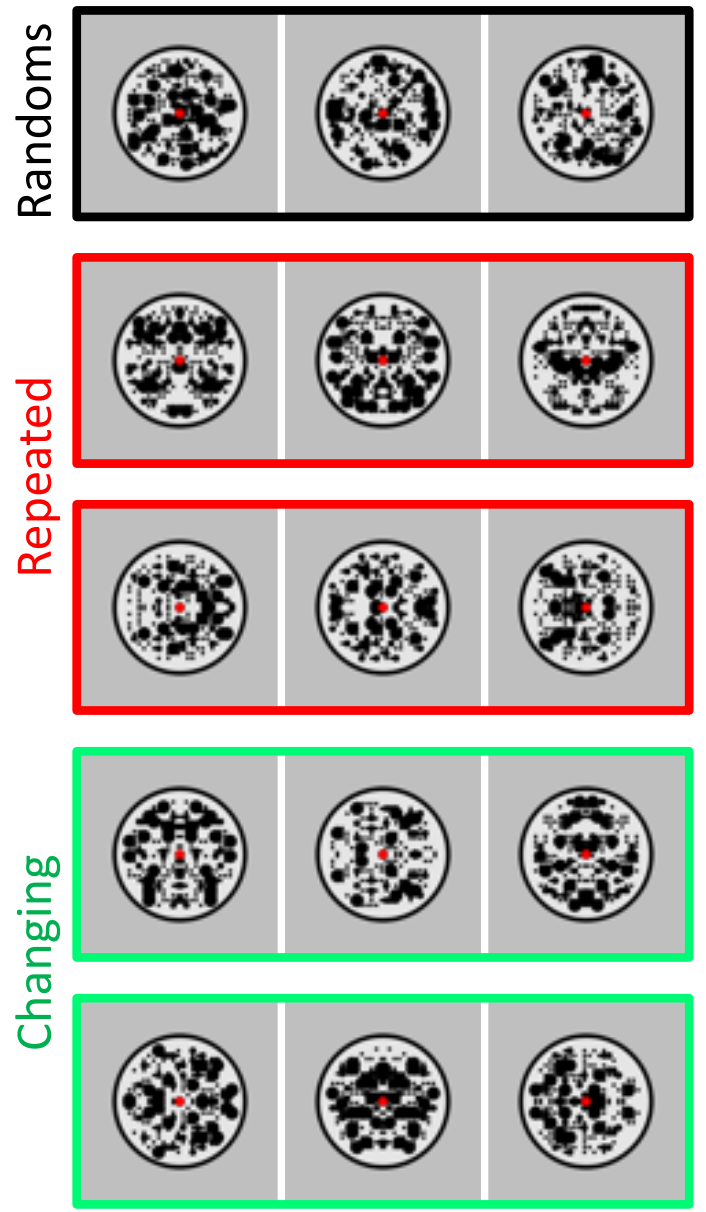

Simple
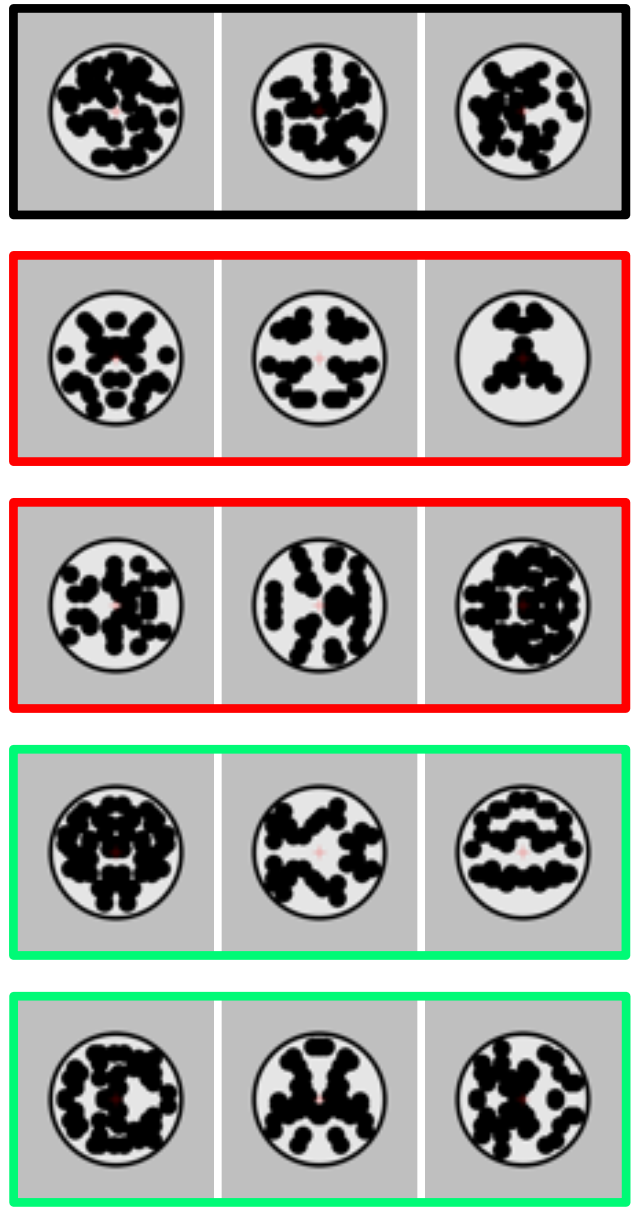

Figure 9. Experiment 4 stimuli. 24 participants were presented with complex patterns (left column) and another 24 were presented with simple patterns (right column).

\section{Experiment 4 results}

Results are shown in Figure 10. All 6 conditions generated a significant SPN $(p<.001)$. Unlike Experiment 3, there was an SPN priming effect in both repeated and changing orientations conditions. A mixed ANOVA revealed a significant main effect of Sequence position ( $F(1.706$, 78.463 $\left.)=8.981, p=.001, \eta_{p}^{2}=.163\right)$, linear contrast $\left(F(1,46)=11.396, p=.002, \eta_{p}^{2}=.199\right)$ but no other effects or interactions $(p>.118)$. Mass univariate analysis confirmed the universal SPN priming in Experiment 4 (supplementary materials 1) 


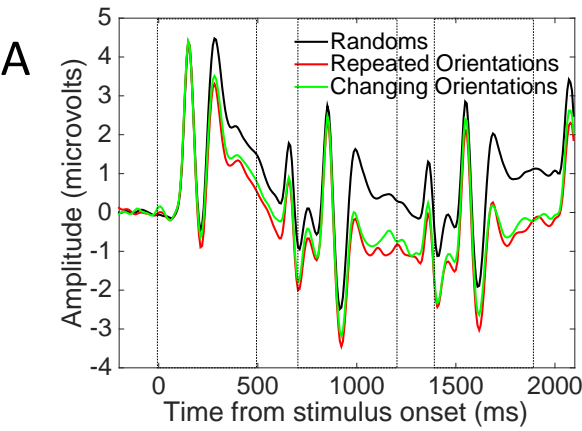

B
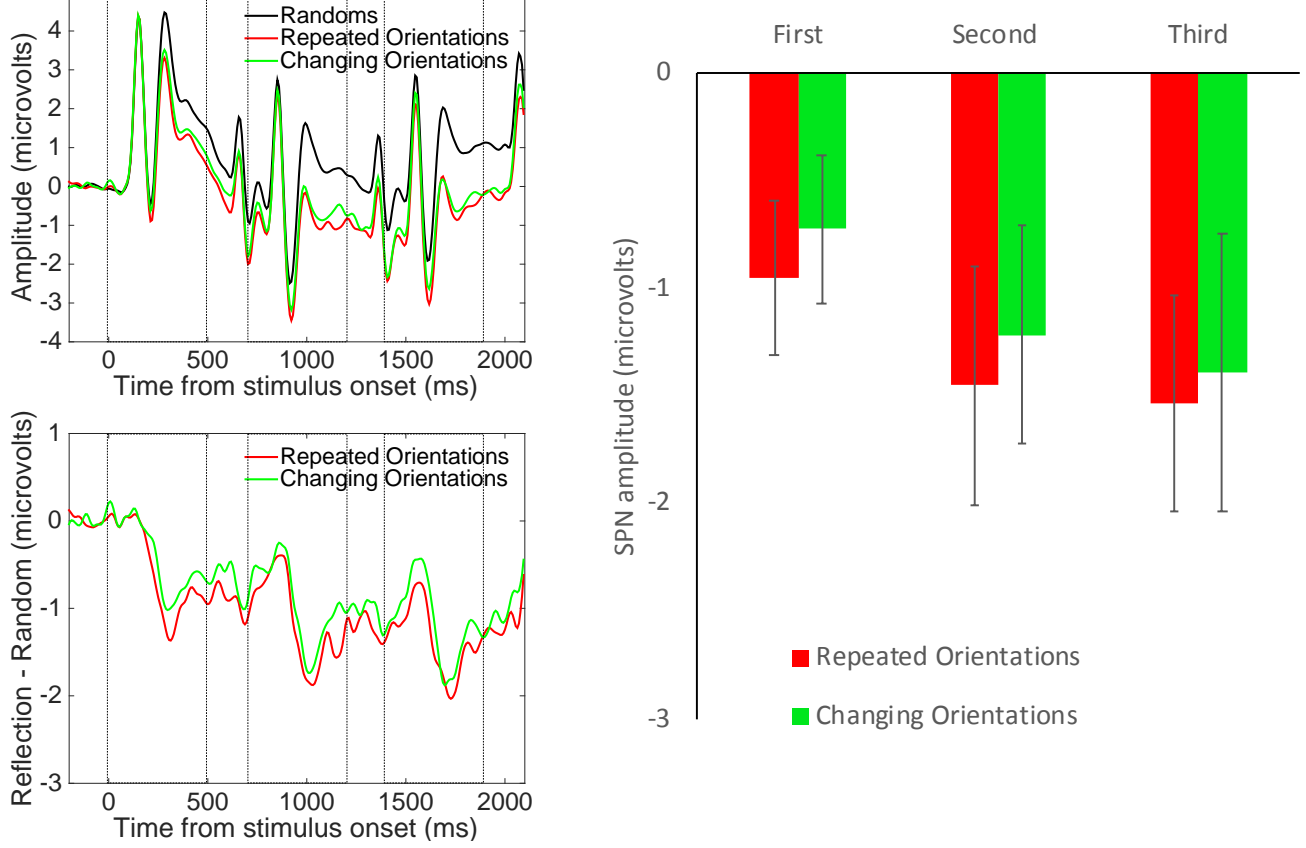

Figure 10. Experiment 4 results. Note SPN priming in both repeated and changing orientation conditions.

A source dipole model comprising of two bilateral sources within the extrastriate regions explained 76.7\% of variance. Both left ECD1 (Brodmann area 19; $-x=-24.3, y=-64.7$, $z=-8.1$ ) and right ECD2 (Brodmann area 19; $-x=24.3, y=-64.7, z=-8.1$ ) were located within the fusiform gyrus. The final model is detailed in Figure $11 \mathrm{~A}$, and the resulting source waveforms are illustrated in Figure 11B. It can be seen that the SPN priming effect was found in both hemispheres, an in both repeated and changing orientation conditions. This was confirmed by three-way repeated measures ANOVA [Sequence position (first, second, third) X Sequence type (Repeated orientations, Changing orientations) X Hemisphere (left (ECD1); right $(E C D 2)]$, which revealed a main effect of Sequence position $(F(1.524,71.603)=11.362$, $\left.p<.001, \eta_{p}^{2}=.195\right)$, but no other effects or interactions $(p>0.229)$. 


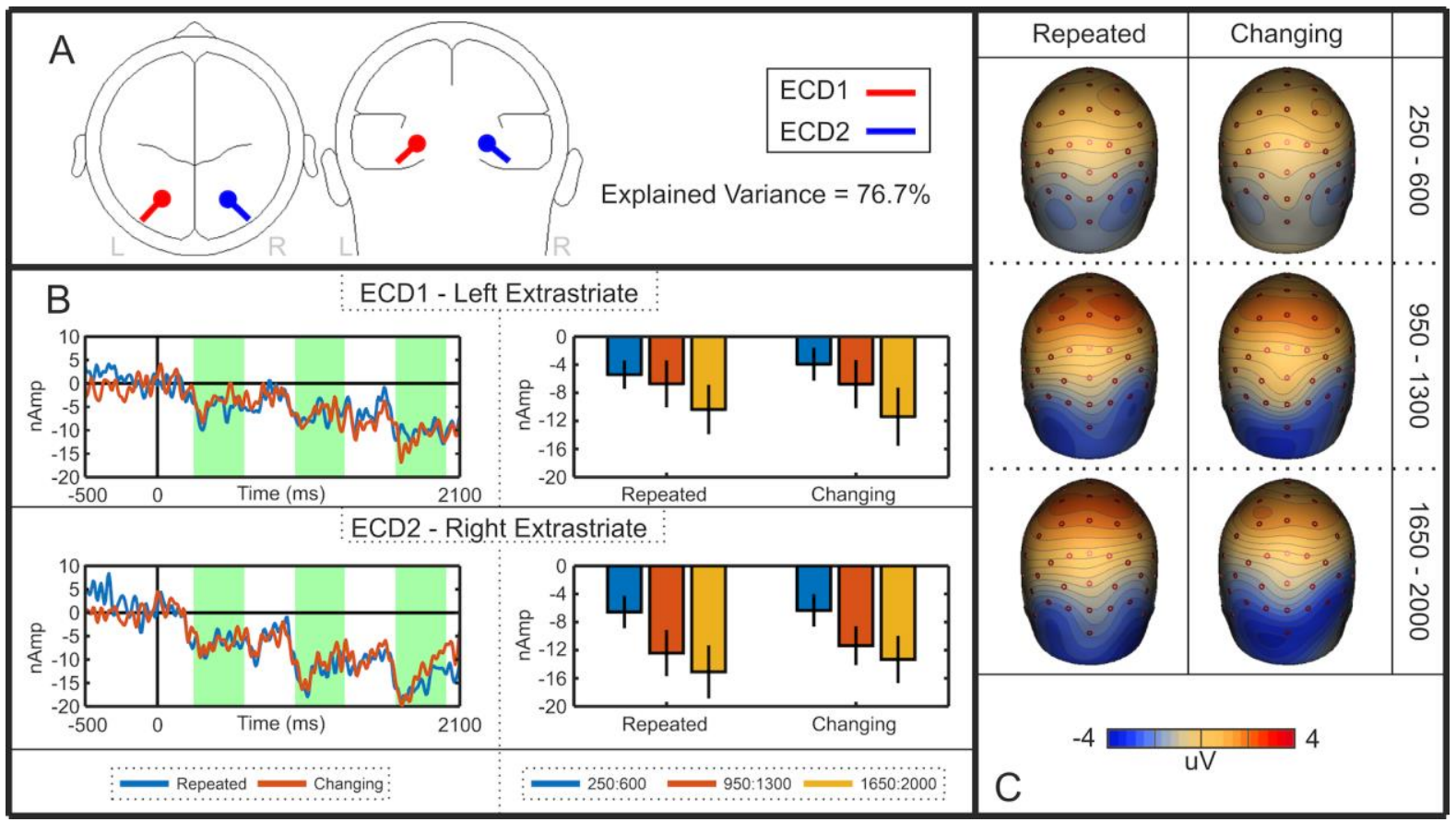

Figure 11. Experiment 4 source dipole model. Conventions are the same as Figure 3, albeit with different $Y$ axis scales. Note the ubiquitous SPN priming effect in the left and right extrastriate source waveforms.

\section{Experiment 4 Discussion}

Experiment 4 provided the first clear example of SPN priming transfer. Namely, SPN priming transferred between vertical and horizontal orientations. While we cannot be sure that transfer would be replicated with other orthogonal axis pairs (e.g. 45 and 135 degrees), Experiment 4 suggests that vertical and horizontal axes are perceptually coupled (Treder et al., 2011).

\section{Experiment 5: Consistent and changing regularities}

Experiment 5 examined independence of reflection and rotational symmetry. We used 90 degree rotation and 1 fold reflection, because these were approximately matched for salience in a pilot behavioural study with 10 participants. The consistent regularity condition involved three reflections or three rotations. The changing regularity condition involved sequences 
where reflection and rotation alternated (Figure 12). Despite the results of Experiment 4, we expected reflection and rotation to be independent in Experiment 5.

\section{Experiment 5 procedure}

Another 48 participants were recruited (age 16-52, mean age 25, 12 male, 6 left handed). As usual, there were 24 participants in the simple pattern condition and 24 in the complex pattern conditions (Figure 12). There were 30 blocks of 18 trials. There were 240 random patterns. There were 60 repeats of each regular sequence type and 60 additional oddball trials (11.1\%). On average participants gave the correct answer on $98 \%$ of trials.
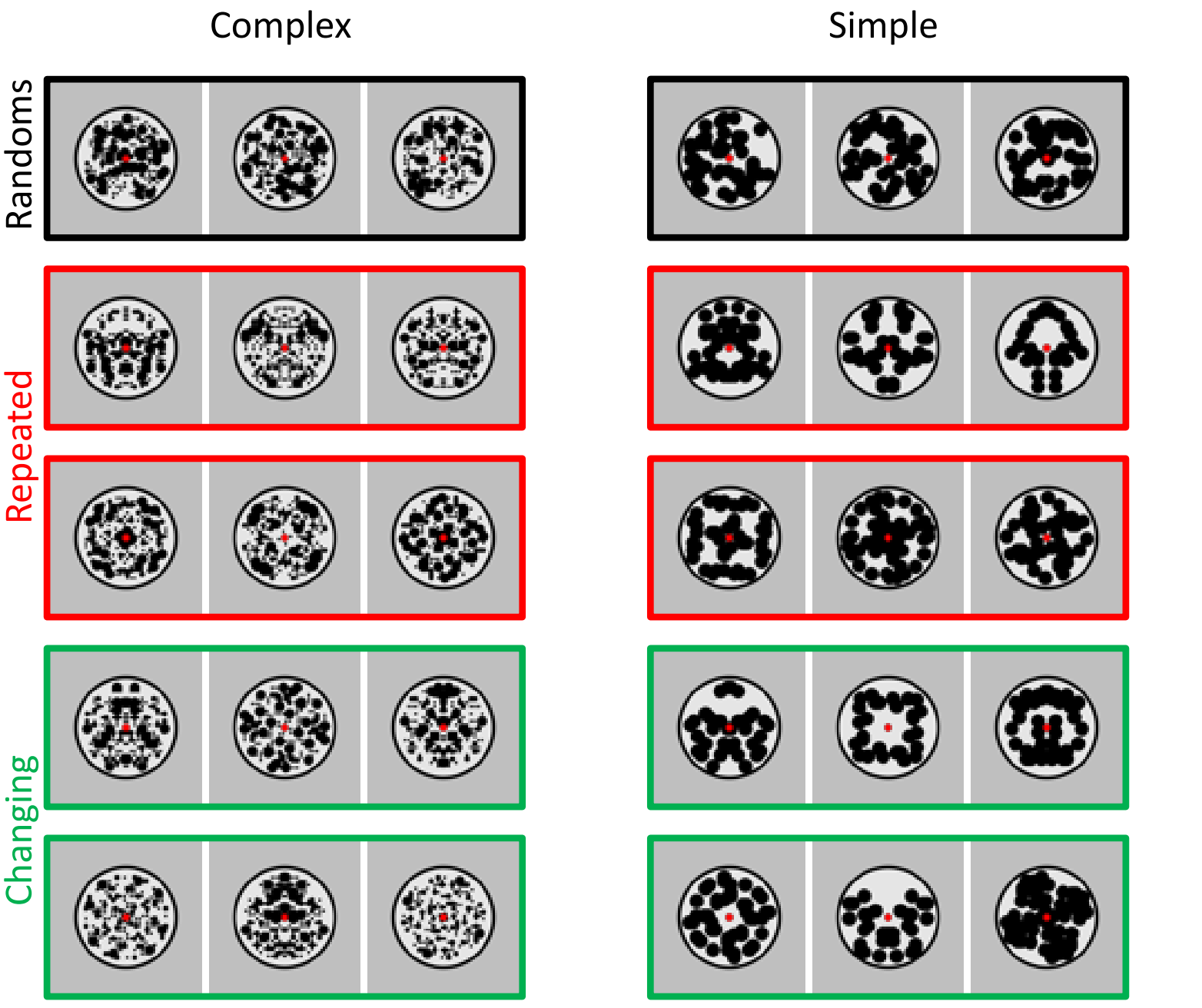
Figure 12. Experiment 5 stimuli. 24 participants were presented with complex patterns (left column) and another 24 were presented with simple patterns (right column).

\section{Experiment 5 results}

Results are shown in Figure 13. All 6 conditions generated a significant SPN $(p<.001)$. There was an SPN priming effect in both repeated and changing regularity conditions. A mixed ANOVA revealed a significant main effect of Sequence position only $(F(1.383,63.602)=$ 18.765, $p<.001, \eta_{p}{ }^{2}=.290$, linear contrast $\left.F(1,46)=21.848, p<.001, \eta_{p}{ }^{2}=.322\right)$. There were no other effects or interactions $(p>.114)$. However, Unlike Experiments 1-4, mass univariate analysis did not simply re-illustrate the effects at posterior electrodes (supplementary materials 1$)$.
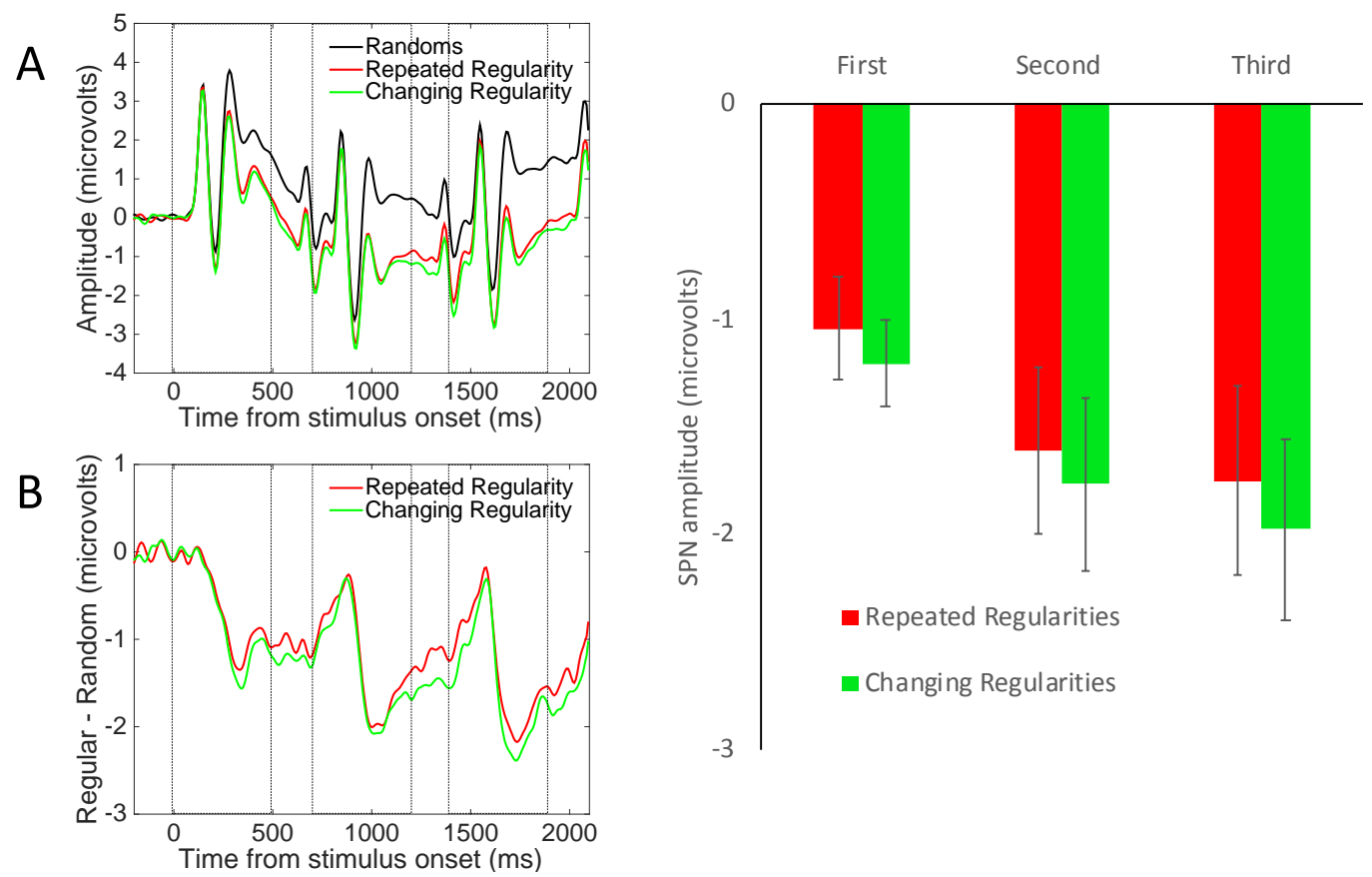

Figure 13. Experiment 5 results. Note SPN priming in both repeated and changing regularity conditions. 
As with Experiment 4, a source dipole model comprising of two bilateral sources within the extrastriate regions explained $90.3 \%$ of variance. Both left ECD1 (Brodmann area $19 ;-x=-25.6, y=-60.8, z=-10.2$ ) and right ECD2 (Brodmann area 19; $-x=25.6, y=-60.8, z$ $=-10.2$ ) were located within the fusiform gyrus. The final model is detailed in Figure 14. The SPN priming effect was evident in both extrastriate dipoles. This replicates the source waveform analysis in Experiments 1 and 4. However, there was one unexpected effect: the SPN was selectively attenuated in the left hemisphere during the changing regularity condition.

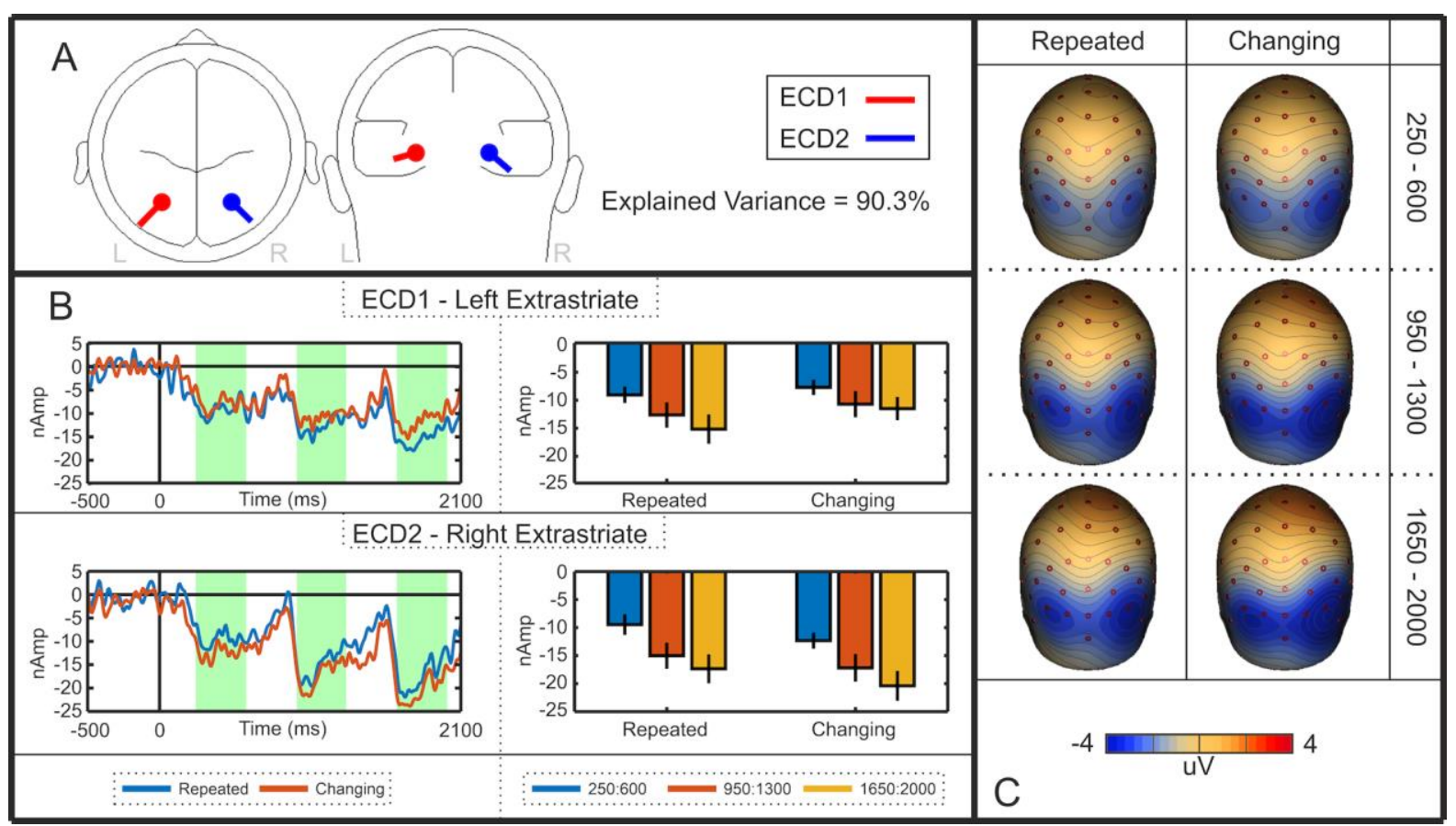

Figure 14. Experiment 5 source dipole model. Note the ubiquitous SPN priming effect in these source waveforms, and right lateralization in the changing regularity condition.

We performed a three-way repeated measures ANOVA [Sequence position (first, second, third) X Sequence type (repeated regularity; changing regularity) X Hemisphere (left (ECD1); right $(E C D 2)]$. There was a main effect of Sequence position $(F(1.553,72.996)=16.257$, 
$\left.p<.001, \eta_{p}^{2}=.257\right)$ and Hemisphere $\left(F(1,47)=4.375, p=.042, \eta_{p}^{2}=.085\right)$. Although there was no main effect of Sequence type $(F<1)$. There was an unexpected interaction between Sequence type and Hemisphere $\left(F(1,47)=4.642, p=.036, \eta_{p}^{2}=.090\right)$ because the SPN was right lateralized in the changing regularity condition $\left(F(1,47)=9.184, p=.004, \eta_{p}{ }^{2}=.163\right)$ but not in in the repeated regularity condition $(F<1)$. There were no other effects or interactions $(p>0.207)$.

\section{Experiment 5 discussion}

ERP results of Experiment 5 were surprising. There was an SPN priming effect for repeated regularities, and also when regularity alternated between reflection and rotation. This suggests some overlap between the way reflection and rotation are coded in the visual system. The traditional ERP analysis was straightforward, however, source dipole analysis showed that SPN priming transfer was limited to the right hemisphere, and mass univariate analysis did not show clear SPN priming in the changing regularity condition (supplementary materials 1). Nevertheless, the balance of evidence suggests that SPN priming at least partially transfers from reflection to rotation and vice versa.

\section{General Discussion}

In Experiment 1, we presented triplets of patterns and found that SPN amplitude increased from presentation $1>2>3$ when different exemplars were used. This was a case of repetition enhancement rather than repetition suppression, within the bilateral extrastriate symmetry network.

Our subsequent Experiments exploited the SPN priming effect to test the independence of extrastriate regularity representations. Experiment 2 provided evidence of 
SPN priming within one hemisphere, and no hint of transfer between hemispheres. Experiment 3 found SPN priming when orientation was consistent, but not when it changed unpredictably. These two experiments thus suggest regularity codes are functionally independent. However, Experiment 4 found that SPN priming survived alternation between vertical and horizontal axis orientations. Finally, Experiment 5 found that SPN priming transferred between reflection and rotation. These two experiments suggest regularity codes partially overlap, at least when the regularities are presented at the same retinal location.

Some previous work has also addressed this topic. Psychophysical work has shown that symmetry discrimination is not impaired by noise masks with different spatial frequencies (Julesz \& Chang, 1979) or different orientations (Rainville \& Kingdom, 2000). This suggests that the symmetry representations are built on retinotopic maps of low-level features, and therefore different regularity representations with different low level features do not overlap and perceptually interfere with each other.

In contrast, the current work on SPN priming suggests that disparate regularity codes are not completely independent. There is some cross-talk between neural systems that code reflection and rotation, and between horizontal and vertical reflection, whenever they are presented at the same retinal location. These results suggest more representational overlap than filter models imply. Perhaps there are higher-level visual integration mechanisms with properties not captured by filter models - and priming occurs at this higher level.

\section{Interpretational considerations}

First, we note that experiments where SPN priming was selective were those with a relatively weak SPN signals (Experiments 2 and 3). Perhaps transfer only happens when the symmetry response is above a certain amplitude? 
Second, we note that the SPN priming effect, when present, was always around 0.5 $\mu \mathrm{V}$, whatever the amplitude of the SPNs themselves (e.g. an increase from -2.6 to $-3.3 \mu \mathrm{V}$ in Experiment 1, but only -0.47 to $-1.05 \mu \mathrm{V}$ in Experiment 3). Furthermore, when equivalent current dipole analysis was possible, this lack of scaling was replicated at the source level. Why does the magnitude of the SPN priming effect not increase with the magnitude of SPN itself? This might imply that SPN priming is caused by some non-specific increase in visual alertness which happens whenever the same stimulus type is repeated. However, there was no SPN priming in Experiment 1 when identical patterns were repeated, so this explanation is less plausible.

Third, all ERPs summate at the scalp. Therefore a third repetition-sensitive dipole, outside the bilateral extrastriate symmetry network could cause apparent SPN priming at the sensor level. This could happen even if the extrastriate symmetry network, which generates the SPN, was not itself subject to repetition effects. However, source dipole analysis showed that SPN priming observed at the sensor-level was attributable to increasing activation within the extrastriate cortex. This is an important finding, because the priming effect observed at posterior electrodes would otherwise be ambiguous.

\section{Future work on SPN priming}

Future work could use the SPN priming effect to test many questions about symmetry coding. For instance, people can perceive anti-symmetry, where luminance in symmetrical positions is anti-correlated (e.g. black regions paired with white and white regions paired with black). We know that anti-symmetry generates an SPN, albeit with slightly reduced amplitude (Makin, Rampone, \& Bertamini, 2019; Wright, Mitchell, Dering, \& Gheorghiu, 2018). However, it is uncertain whether symmetry and anti-symmetry are coded by the same 
extrastriate networks. Future work could examine this by testing whether SPN priming generalizes from symmetry to anti-symmetry. Furthermore, one could use the same research strategy to assess whether symmetry is coded independently of luminance, colour or contrast of the elements (also studied by Gheorghiu, Kingdom, Remkes, Li, \& Rainville, 2016). Based on the results of Ouhnana et al. (2013) and the current work, we predict SPN priming to be untouched by variation in these low-level visual features.

In these five experiments participants were not explicitly classifying the patterns as symmetrical or random. It could be that the SPN priming becomes more transferrable when participants attend to regularity. After all, previous studies have shown that the SPN indexes symmetry in the object when participants are attending to regularity, while it only indexes symmetry in the image when participants attend to colour (Makin et al., 2015). Furthermore, when participants attend to regularity, the SPN can be generated by partially occluded symmetry (Rampone, Makin, Tatlidil, \& Bertamini, 2019). It seems that attention to regularity allows the system to go beyond image symmetry, and generate extraretinal, object-level symmetry representations, which show perceptual constancy. We therefore speculate that SPN priming would transfer across changes in location and orientation if participants were attending to regularity and thus working with post-constancy representations.

\section{Conclusions}

SPN priming effect is sometimes selective and it does not survive changes in retinal location or non-orthogonal changes in axis orientation. However, SPN priming is not completely selective. It transfers between horizontal and vertical orientations and between reflection and rotation. We conclude that there are common visual integration mechanisms which can extract different regularities at specific retinal locations. 


\section{General methods}

\section{Participants}

Each of our 5 experiments had 48 participants ( 240 in total). There were 24 participants in the simple pattern condition and 24 in the complex pattern conditions. All participants had normal or corrected to normal vision, except for those Experiment 2 where people with glasses or contact lenses were excluded because it interfered with eye tracking. The study had local ethics committee approval and was conducted in accordance with APA ethics codes.

\section{Apparatus}

All EEG data was collected with a BioSemi Active-2 64 channel EEG system. Electrodes were embedded in an elasticated cap and arranged according to the international 10-20 system. Bipolar HEOG and VEOG channels were monitored for excessive blinking or eye movements. Bandpass filters were set to 0.16 and $100 \mathrm{~Hz}$, and sample rate was $512 \mathrm{~Hz}$.

In all Experiments a chin rest was used for head stabilization. Experiments 1, 3 and 4 were presented on a $40 \times 30 \mathrm{Cm}\left(23 \times 17^{\circ}\right) \mathrm{CRT}$ monitor. Participants were seated $100 \mathrm{~cm}$ back from the monitor. In Experiment 2 participants were seated $57 \mathrm{~cm}$ back from a $53 \times 30^{\circ}$ LCD monitor. In Experiment 5, participants were seated $57 \mathrm{Cm}$ back from a $51 \times 29^{\circ} \mathrm{LCD}$ monitor. All monitors had a refresh rate of $60 \mathrm{~Hz}$. Although the monitors changed between experiments, stimulus size was identical in degrees of visual angle.

All Experiments except Experiment 2 were conducted in an electrically shielded and darkened cubicle. Experiment 2 was conducted on an identical BioSemi EEG recording system, but in a separate eye tracking cubicle that was not electrically shielded. Consequently, the EEG data sometimes included unwanted $50 \mathrm{~Hz}$ mains noise and we set the online low pass 
filter at $30 \mathrm{~Hz}$ to remove this for 2 participants. In Experiment 2, eye position was monitored with a desk mounted Gazepoint $60 \mathrm{~Hz}$ infra-red eye tracker. The experimenter could monitor eye position superimposed on the stimulus in real time, and thus assess whether participants routinely broke fixation. Eye position analysis confirmed that participants were fixating successfully without bias to the symmetrical pattern (see supplementary materials).

\section{Stimulus construction algorithm}

The images were constructed using Python and PsychoPy (Peirce, 2007), and saved as PNG image files. The algorithm for construction of 8 fold reflection or matched random patterns in Experiment 1 is shown in Figure 1. The patterns were based on an implicit grid of 432 cells. This produced 8 segments, each with a central mirror reflection (Figure 15A). The grid was $6.1^{\circ}$ of visual angle in diameter, and the outer black ring was $7.2^{\circ}$ in diameter.

Next half of each segment was occupied with dots in a probabilistic fashion (Figure 15B). For complex patterns, each cell had a $50 \%$ probability of occupation. When occupied, small $\left(0.18^{\circ}\right.$ diameter $)$, medium $\left(0.36^{\circ}\right.$ diameter $)$ or large $\left(0.72^{\circ}\right.$ diameter $)$ dots were chosen at random with from a distribution with a ratio of 6:2:1. In other words, on average, there were 3 times as many small dots as medium, and twice as many medium as large (again this was probabilistic, so it would be possible, albeit extremely unlikely, for all dots to be the same size). For regular patterns, the arrangement was mirrored in each segment, and all segments were identical (Figure 15C). For random patterns, each segment was independent, and the dots on each side were not mirrored. The average number of small, medium and large dots was the same in the regular and random patterns. However, the dot-size variability between patterns was higher for regular patterns because only one segment was original, so any statistically usual properties became like frozen accidents in the other segments. 
For simple patterns, the construction algorithm and implicit grid were the same, the probability of occupation was set at $10 \%$, and all dots were $1^{\circ}$ diameter. This results in stimuli with a greater distribution of energy at lower spatial frequencies, larger 'blobby' substructures, and reduced coastline of black regions.

Stimulus construction was the same for Experiments 2-5, except the implicit grid was changed. In Experiment 2 the images were resized so the black ring was $5^{\circ}$ in diameter, but the proportional sizes of elements were the same as all other experiments. One-fold reflections was based on an implicit grid with a single vertical seam, and 90 degree rotations had 4 seams. In Experiments 3 and 4, the images were rotated after generation. In Experiment 5 were 120 random triplets based on the $1 \mathrm{~F}$ grid, and 120 based on the $4 \mathrm{~F}$ grid. This made no discernible difference to the appearance of the random patterns. All stimuli and image construction algorithms are available on Open Science Framework, and are freely available for other researchers: https://osf.io/2yjus/

The required number of regular and random images were generated in advance, so no pattern was ever presented twice. In each experiment all participants were shown the same set of images. However, images were shuffled so that they played different roles for each participant. For instance, a particular reflection might appear as the first in a reflection triplet for one participant, as the third pattern in a RandRandRef sequence for another participant. This abolished any potential effects of chance stimulus features on our results. 


\section{A) Grid of locations}

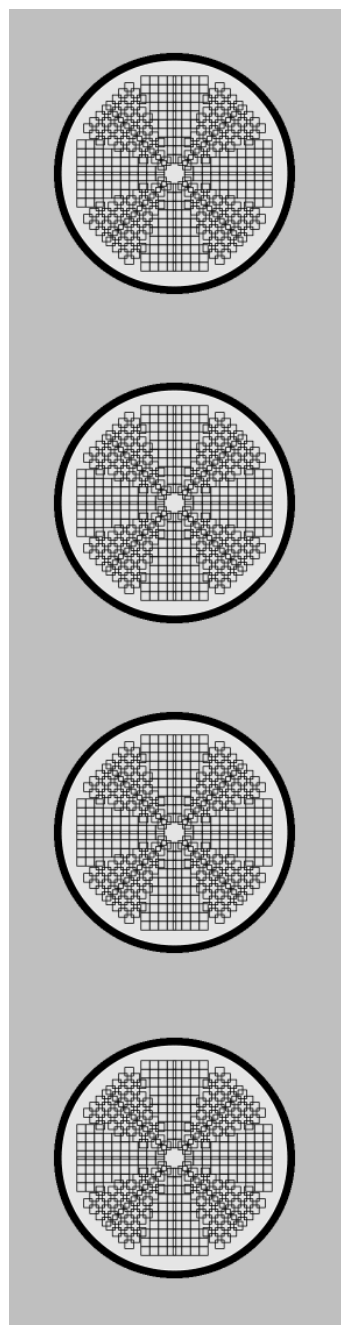

B) First half occupied

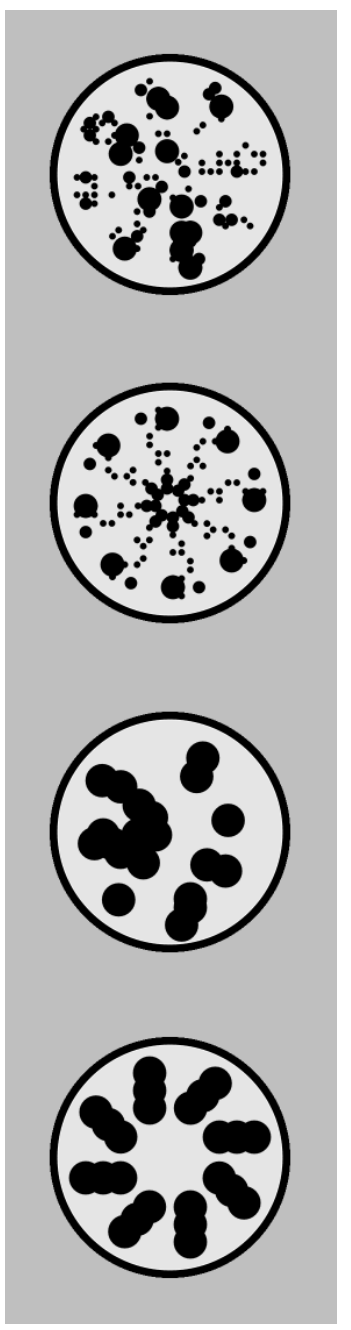

C) Second half occupied or mirrored

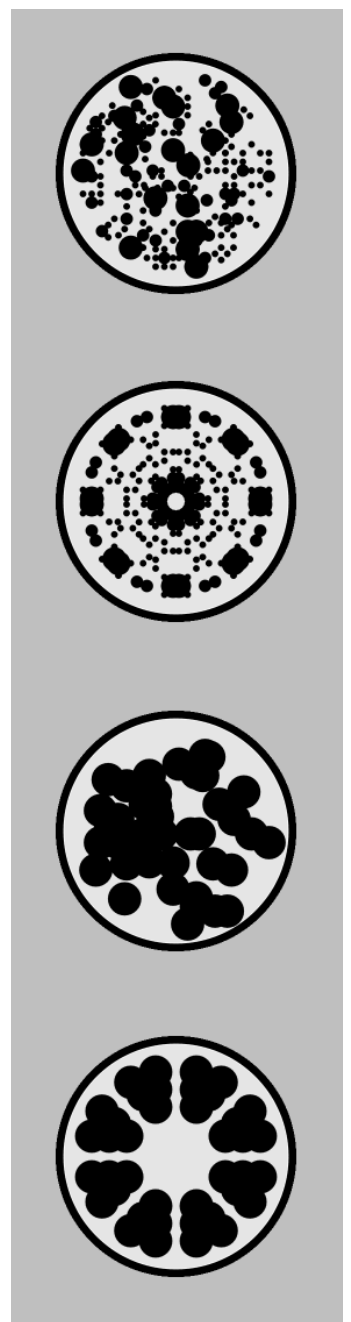

Figure 15. Stimulus construction steps. A) An in implicit grid of possible locations was created. B) The grid was probabilistically occupied with black dots. For complex stimuli, the black dots were small, medium or large (top two rows). For simple stimuli, all black dots were large (lower two rows). C) For random patterns each segment half was occupied independently. For reflectional pattern, each half was mirrored.

\section{Basic ERP analysis}

EEG analysis was based on our previous studies. EEG data was analysed offline using eeglab 13.4 functions in Matlab 2014b (Delorme \& Makeig, 2004). Data from the 64 scalp electrodes was re-referenced to the scalp average, and low pass filtered at $25 \mathrm{~Hz}$. We then 
downsampled to $128 \mathrm{~Hz}$ to reduced file size, and segmented the data in epochs from -0.5 to +2.1 seconds around the onset of the first pattern.

Independent Components Analysis (Jung et al., 2000) then used to remove blink and other high amplitude artefacts. The number of ICA components removed was as follows: Experiment 1: $\operatorname{mean}=9.75, \min =0, \max =20$, Experiment 2 mean $=12.17, \min =4, \max =$ 22, Experiment 3: $\operatorname{mean}=11.17, \min =2, \max =21$. Experiment 4 mean $=9.43, \min =2, \max$ $=13:$ Experiment 5: mean $=9.06, \min 2, \max =21$.

After cleaning with ICA, epochs where amplitude exceeded $+/-100 \mu \mathrm{V}$ at any electrode were excluded. Mean exclusion rates were as follows: Experiment $1 ; 11-14 \%$, Experiment 2: 14-15\%, Experiment 3: 14-15\%, Experiment 4: 15-16\%, Experiment 5: 9-10\%.

The remaining trials in each condition were averaged to provide the data for ERP analysis with mixed ANOVA. The Greenhouse-Geisser correction factor was used whenever the assumption of sphericity was violated $(p<.05)$. Across the 5 Experiments we analysed 33 SPN difference waves (i.e. each bar in the results figures). Only 1/33 SPN waves violated the assumption of normality according to the Kolmogorov-Smirnov test $(p<.05)$. This replicates previous experiments, where individual participant SPN amplitudes are usually normally distributed around the grand average. ERP data used for plotting and statistical analysis is also available on OSF https://osf.io/2yjus/

\section{Source dipole modelling}

In order to investigate the spatiotemporal dynamics of SPN priming, a source dipole model was constructed in BESA v. 7.0 (MEGIS GmbH, Munich, Germany) for each experiment. For the greatest accuracy of source localization, it is necessary to utilize data with a large signalto-noise ratio. To achieve this, difference waves (symmetry - random) for each condition 
were averaged to produce a single grand-average waveform representing symmetry-specific responses. This was done for each experiment individually.

The protocol for producing an appropriate source dipole model required that equivalent current dipoles (ECDs) were fitted to describe the 3-dimensional source currents in the regions contributing predominantly to the data. Principle component analysis (PCA) was first used to identify an appropriate number of ECDs to fit. Since previous studies have identified bilateral extrastriate cortices as being the primary generators of symmetry specific neural activity (Keefe et al., 2018; Sasaki et al., 2005; Van Meel et al., 2019) two ECDs were first inserted in the bilateral extrastriate regions. Following the insertion of these two ECDs, residual variance was used as a tool for indicating the sufficiency of the model. The ECD fitting procedure required waveforms with a large signal-to-noise ratio and intervals with a strong cortical response. Therefore, the weak SPN observed in Experiment 2 and 3 could not be accurately modelled. Since data across experiment 1,4 and 5 were sufficiently explained by bilateral extrastriate ECDs, no further fitting of ECDs were required.

Classical LORETA analysis recursively applied (CLARA), which is an iterative application of the LORETA algorithm (Pascual-Marqui et al., 1994) was used to confirm and adjust the locations of the ECDs in the final model. Following the fitting of the ECD locations, the orientation of the ECDs then had to be determined. Since there are differences between individuals regarding gyral anatomy in the brain, ECD orientation was determined on a subject-by-subject basis, but with fixed location between subjects, based on the entire corresponding grand average difference waveform. A 4-shell ellipsoid head volume conductor model was employed using the following conductivities $(\mathrm{S} / \mathrm{m}=$ Siemens per meter): Brain = $.33 \mathrm{~S} / \mathrm{m}$; Scalp $=.33 \mathrm{~S} / \mathrm{m}$; Bone $=.0042 \mathrm{~S} / \mathrm{m}$, Cerebrospinal Fluid $=1 \mathrm{~S} / \mathrm{m}$. Source waveforms 
for each experiment and condition were exported and analyzed using repeated-measures ANOVAs.

\section{Acknowledgments}

This project was part funded by an ESRC grant award to Marco Bertamini (ES/K000187/1) and part funded by an ESRC grant awarded to Alexis Makin (ES/S014691/1). We would like to thank the University of Liverpool students who helped with data collection: Husnara Ali, Jamie Benyon, Megan Bowen, Faye Clancy, Rebecca Davies, Scarlett Griffin, Adie Howarth, Rebecca Rogers, Nathan Rooney. We would also like to thank the Internship students on summer placement from the University of Calgary: Aya Ebdalla and Emily Drake (2018) and Haya Bakour and Jessica Hsieh (2019).

\section{References}

Barlow, H. B., \& Reeves, B. C. (1979). Versatility and absolute efficiency of detecting mirror symmetry in random dot displays. Vision Research, 19(7), 783-793. https://doi.org/10.1016/0042-6989(79)90154-8

Bauer, C., Yazzolino, L., Hirsch, G., Cattaneo, Z., Vecchi, T., \& Merabet, L. B. (2015). Neural correlates associated with superior tactile symmetry perception in the early blind. Cortex, 63, 104-117. https://doi.org/10.1016/J.CORTEX.2014.08.003

Bertamini, M., \& Makin, A. D. J. (2014). Brain activity in response to visual symmetry. Symmetry, 6, 975-996. https://doi.org/10.3390/sym6040975

Bertamini, M., Silvanto, J., Norcia, A. M., Makin, A. D. J., \& Wagemans, J. (2018). The neural basis of visual symmetry and its role in mid- and high-level visual processing. Annals of the New York Academy of Sciences, 1426(1), 111-126. https://doi.org/10.1111/nyas.13667

Bona, S., Cattaneo, Z., \& Silvanto, J. (2015). The causal role of the occipital face area (OFA) and lateral occipital (LO) cortex in symmetry perception. The Journal of Neuroscience, 35(2), 731-738. https://doi.org/10.1523/JNEUROSCl.3733-14.2015

Cattaneo, Z. (2017). The neural basis of mirror symmetry detection: a review. Journal of Cognitive Psychology, 29(3), 259-268. https://doi.org/10.1080/20445911.2016.1271804

Cattaneo, Z., Mattavelli, G., Papagno, C., Herbert, A., \& Silvanto, J. (2011). The role of the human extrastriate visual cortex in mirror symmetry discrimination: A TMS-adaptation study. Brain and Cognition, 77(1), 120-127. https://doi.org/10.1016/j.bandc.2011.04.007

Chen, C. C., Kao, K. L. C., \& Tyler, C. W. (2007). Face configuration processing in the human brain: The role of symmetry. Cerebral Cortex, 17(6), 1423-1432. https://doi.org/10.1093/cercor/bhl054

Clark, A. (2013). Whatever next? Predictive brains, situated agents, and the future of cognitive science. Behavioral and Brain Sciences, 36(3), 181-204. https://doi.org/10.1017/s0140525×12000477

Dakin, S. C., \& Hess, R. F. (1997). The spatial mechanisms mediating symmetry perception. 
Vision Research, 37(20), 2915-2930. https://doi.org/10.1016/S0042-6989(97)00031-X

Delorme, A., \& Makeig, S. (2004). EEGLAB: an open source toolbox for analysis of single-trial EEG dynamics including independent component analysis. Journal of Neuroscience Methods, 134(1), 9-21. https://doi.org/10.1016/j.jneumeth.2003.10.009

Gheorghiu, E., Kingdom, F. A. A., Remkes, A., Li, H.-C. O., \& Rainville, S. (2016). The role of color and attention-to-color in mirror-symmetry perception. Scientific Reports, 6 , 29287. https://doi.org/10.1038/srep29287

Grammer, K., Fink, B., Møller, A. P., \& Thornhill, R. (2003). Darwinian aesthetics: sexual selection and the biology of beauty. Biological Reviews, 78(3), 385-407. https://doi.org/10.1017/s1464793102006085

Grill-Spector, K., Henson, R., \& Martin, A. (2006). Repetition and the brain: neural models of stimulus-specific effects. Trends in Cognitive Sciences, 10(1), 14-23. https://doi.org/10.1016/J.TICS.2005.11.006

Henson, R., Shallice, T., Science, R. D.-, \& 2000, U. (2000). Neuroimaging evidence for dissociable forms of repetition priming. Science, 287(5456), 1269-1272.

Höfel, L., \& Jacobsen, T. (2007). Electrophysiological indices of processing aesthetics: Spontaneous or intentional processes? International Journal of Psychophysiology, 65(1), 20-31. https://doi.org/10.1016/j.ijpsycho.2007.02.007

Jacobsen, T., \& Höfel, L. (2003). Descriptive and evaluative judgment processes: Behavioral and electrophysiological indices of processing symmetry and aesthetics. Cognitive Affective \& Behavioral Neuroscience, 3(4), 289-299. https://doi.org/10.3758/CABN.3.4.289

Julesz, B., \& Chang, J.-J. (1979). Symmetry Perception and Spatial-Frequency Channels. Perception, 8(6), 711-718. https://doi.org/10.1068/p080711

Jung, T. P., Makeig, S., Humphries, C., Lee, T. W., McKeown, M. J., Iragui, V., \& Sejnowski, T. J. (2000). Removing electroencephalographic artifacts by blind source separation. Psychophysiology, 37(2), 163-178.

Keefe, B. D., Gouws, A. D., Sheldon, A. A., Vernon, R. J. W., Lawrence, S. J. D., McKeefry, D. J., ... Morland, A. B. (2018). Emergence of symmetry selectivity in the visual areas of the human brain: $\mathrm{fMRI}$ responses to symmetry presented in both frontoparallel and slanted planes. Human Brain Mapping. https://doi.org/10.1002/hbm.24211

Kim, J. G., Biederman, I., Lescroart, M. D., \& Hayworth, K. J. (2009). Adaptation to objects in the lateral occipital complex (LOC): Shape or semantics? Vision Research, 49(18), 22972305. https://doi.org/10.1016/J.VISRES.2009.06.020

Knill, D. C., \& Pouget, A. (2004). The Bayesian brain: the role of uncertainty in neural coding and computation. Trends in Neurosciences, 27(12), 712-719. https://doi.org/10.1016/j.tins.2004.10.007

Kohler, P. ., Clarke, A., Yakovleva, A., Liu, Y., \& Norcia, A. M. (2016). Representation of maximally regular textures in human visual cortex. The Journal of Neuroscience, 36(3), 714-729. https://doi.org/10.1523/JNEUROSCI.2962-15.2016

Kohler, P. J., Cottereau, B. R., \& Norcia, A. M. (2018). Dynamics of perceptual decisions about symmetry in visual cortex. Neurolmage, 167, 316-330. https://doi.org/10.1016/J.NEUROIMAGE.2017.11.051

Kourtzi, Z., \& Kanwisher, N. (2001). Representation of perceived object shape by the human lateral occipital complex. Science, 293(5534), 1506-1509. https://doi.org/10.1126/science.1061133

Mach, E. (1886). The analysis of sensations and the relation of the phsysical to the psychical. 
New York: Dover.

Machilsen, B., Pauwels, M., \& Wagemans, J. (2009). The role of vertical mirror symmetry in visual shape detection. Journal of Vision, 9(12), 1-11. https://doi.org/11 10.1167/9.12.11

Makin, A. D. J., Rampone, G., \& Bertamini, M. (2015). Conditions for view invariance in the neural response to symmetry. Psychophysiology, 52(4), 532-543. https://doi.org/DOI: 10.1111/psyp.12365

Makin, A. D. J., Rampone, G., \& Bertamini, M. (2019). Symmetric patterns with different luminance polarity (anti-symmetry) generate an automatic response in extrastriate cortex. European Journal of Neuroscience, ejn.14579. https://doi.org/10.1111/ejn.14579

Makin, A. D. J., Rampone, G., Morris, A., \& Bertamini, M. (2020). The Formation of Symmetrical Gestalts Is Task Independent, but Can Be Enhanced by Active Regularity Discrimination. Journal of Cognitive Neuroscience, 32(2), 353-366. https://doi.org/10.1162/jocn_a_01485

Makin, A. D. J., Rampone, G., Pecchinenda, A., \& Bertamini, M. (2013). Electrophysiological responses to visuospatial regularity. Psychophysiology, 50, 1045-1055. https://doi.org/10.1111/psyp.12082

Makin, A. D. J., Rampone, G., Wright, A., Martinovic, J., \& Bertamini, M. (2014). Visual symmetry in objects and gaps. Journal of Vision, 14(3), 1-12. https://doi.org/10.1167/14.3.12.

Makin, A. D. J., Wilton, M. M., Pecchinenda, A., \& Bertamini, M. (2012). Symmetry perception and affective responses: A combined EEG/EMG study. Neuropsychologia, 50(14), 3250-3261. https://doi.org/10.1016/j.neuropsychologia.2012.10.003

Makin, A. D. J., Wright, D., Rampone, G., Palumbo, L., Guest, M., Sheehan, R., ... Bertamini, M. (2016). An electrophysiological index of perceptual goodness. Cerebral Cortex, 26, 4416-4434. https://doi.org/doi: 10.1093/cercor/bhw255

Mancini, S., Sally, S. L., \& Gurnsey, R. (2005). Detection of symmetry and anti-symmetry. Vision Research, 45(16), 2145-2160. https://doi.org/10.1016/j.visres.2005.02.004

Martinovic, J., Jennings, B. J., Makin, A. D. J., Bertamini, M., \& Angelescu, I. (2018). Symmetry perception for patterns defined by color and luminance. Journal of Vision, 18(8), 1-24. https://doi.org/10.1167/18.8.4

Miller, E. K., Li, L., \& Desimone, R. (1991). A neural mechanism for working and recognition memory in inferior temporal cortex. Science, 254(5036), 1377-1379. https://doi.org/10.1126/science.1962197

Milner, A. D., Perrett, D. I., Johnston, R. S., Benson, P. J., Jordan, T. R., Heeley, D. W., ... Davidson, D. L. W. (1991). Perception and action in "visual form agnosia." Brain, 114, 405-428.

Møller, A. P. (1992). Female Swallow Preference For Symmetrical Male Sexual Ornaments. Nature, 357(6375), 238-240. https://doi.org/10.1038/357238a0

Morgan, M. J., Mareschal, I., Chubb, C., \& Solomon, J. A. (2012). Perceived pattern regularity computed as a summary statistic: implications for camouflage. Proceedings. Biological Sciences, 279(1739), 2754-2760. https://doi.org/10.1098/rspb.2011.2645

Nieuwenhuis, S., Forstmann, B. U., \& Wagenmakers, E. J. (2011). Erroneous analyses of interactions in neuroscience: a problem of significance. Nature Neuroscience, 14(9), 1105-1107. https://doi.org/10.1038/nn.2886

Niimi, R., Watanabe, K., \& Yokosawa, K. (2008). The dynamic-stimulus advantage of visual 
symmetry perception. Psychological Research, 72(5), 567-579. https://doi.org/10.1007/s00426-008-0133-y

Ouhnana, M., Bell, J., Solomon, J. A., \& Kingdom, F. A. A. (2013). Aftereffect of perceived regularity. Journal of Vision, 13(8), 1-13. https://doi.org/10.1167/13.8.18

Palumbo, L., Bertamini, M., \& Makin, A. (2015). Scaling of the extrastriate neural response to symmetry. Vision Research, 117, 1-8. https://doi.org/10.1016/j.visres.2015.10.002

Pascual-Marqui, R. D., Michel, C. M., \& Lehmann, D. (1994). Low-Resolution electromagnetic tomography - A new method for localizing electrical activity in the brain. International Journal of Psychophysiology, 18(1), 49-65. https://doi.org/10.1016/01678760(84)90014-x

Peirce, J. W. (2007). PsychoPy - Psychophysics software in Python. Journal of Neuroscience Methods, 162(1-2), 8-13. https://doi.org/10.1016/j.jneumeth.2006.11.017

Poirier, F., \& Wilson, H. R. (2010). A biologically plausible model of human shape symmetry perception. Journal of Vision, 10(1). https://doi.org/910.1167/10.1.9

Rainville, S. J. M., \& Kingdom, F. A. A. (2000). The functional role of oriented spatial filters in the perception of mirror symmetry - psychophysics and modeling. Vision Research, 40(19), 2621-2644. https://doi.org/10.1016/S0042-6989(00)00110-3

Rampone, G., Makin, A. D. J., Tatlidil, S., \& Bertamini, M. (2019). Representation of symmetry in the extrastriate visual cortex from temporal integration of parts: An EEG/ERP study. Neurolmage, 193, 214-230. https://doi.org/10.1016/J.NEUROIMAGE.2019.03.007

Rao, R. P. N., \& Ballard, D. H. (1999). Predictive coding in the visual cortex: a functional interpretation of some extra-classical receptive-field effects. Nature Neuroscience, 2(1), 79-87. https://doi.org/10.1038/4580

Rhodes, G., Proffitt, F., Grady, J. M., \& Sumich, A. (1998). Facial symmetry and the perception of beauty. Psychonomic Bulletin \& Review, 5(4), 659-669. https://doi.org/10.3758/BF03208842

Sasaki, Y., Vanduffel, W., Knutsen, T., Tyler, C. W., \& Tootell, R. (2005). Symmetry activates extrastriate visual cortex in human and nonhuman primates. Proceedings of the National Academy of Sciences of the United States of America, 102(8), 3159-3163. https://doi.org/10.1073/pnas.0500319102

Sharman, R. J., \& Gheorghiu, E. (2017). The role of motion and number of element locations in mirror symmetry perception. Scientific Reports, 7, 45679. https://doi.org/10.1038/srep45679

Treder, M. S. (2010). Behind the looking glass: a review on human symmetry perception. Symmetry, 2, 1510-1543. https://doi.org/10.3390/sym2031510

Treder, M. S., van der Vloed, G., \& van der Helm, P. A. (2011). Interactions between constituent single symmetries in multiple symmetry. Attention, Perception, \& Psychophysics, 73(5), 1487-1502. https://doi.org/10.3758/s13414-011-0115-9

Tyler, C. W., Baseler, H. A., Kontsevich, L. L., Likova, L. T., Wade, A. R., \& Wandell, B. A. (2005). Predominantly extra-retinotopic cortical response to pattern symmetry. Neuroimage, 24(2), 306-314. https://doi.org/10.1016/j.neuroimage.2004.09.018

Tyson-Carr, J., Kokmotou, K., Soto, V., Cook, S., Fallon, N., Giesbrecht, T., \& Stancak, A. (2018). Neural correlates of economic value and valuation context: an event-related potential study. Journal of Neurophysiology, 119(5), 1924-1933. https://doi.org/10.1152/jn.00524.2017

van der Helm, P. A., \& Leeuwenberg, E. L. J. (1996). Goodness of visual regularities: A 
nontransformational approach. Psychological Review, 103(3), 429-456. https://doi.org/10.1037/0033-295x.103.3.429

Van Meel, C., Baeck, A., Gillebert, C. R., Wagemans, J., \& Op de Beeck, H. P. (2019). The representation of symmetry in multi-voxel response patterns and functional connectivity throughout the ventral visual stream. Neurolmage, 191, 216-224. https://doi.org/10.1016/j.neuroimage.2019.02.030

Wagemans, J. (1995). Detection of visual symmetries. Spatial Vision, 9(1), 9-32. https://doi.org/10.1163/156856895X00098

Wenderoth, P. (1994). The salience of vertical symmetry. Perception, 23(2), 221-236. https://doi.org/10.1068/p230221

Wignall, A. E., Heiling, A. M., Cheng, K., \& Herberstein, M. E. (2006). Flower symmetry preferences in honeybees and their crab spider predators. Ethology, 112(5), 510-518. https://doi.org/10.1111/j.1439-0310.2006.01199.x

Wright, D., Makin, A. D. J., \& Bertamini, M. (2017). Electrophysiological responses to symmetry presented in the left or in the right visual hemifield. Cortex, 86, 93-108. https://doi.org/10.1016/j.cortex.2016.11.001

Wright, D., Mitchell, C., Dering, B. R., \& Gheorghiu, E. (2018). Luminance-polarity distribution across the symmetry axis affects the electrophysiological response to symmetry. Neurolmage, 173, 484-497.

https://doi.org/10.1016/J.NEUROIMAGE.2018.02.008

Yamada, Y., Kawabe, T., \& Miyazaki, M. (2013). Pattern randomness aftereffect. Scientific Reports, 3(1), 1-8. https://doi.org/10.1038/srep02906

Yamauchi, T., Cooper, L. A., John Hilton, H., Szerlip, N. J., Chen, H.-C., \& Barnhardt, T. M. (2006). Priming for symmetry detection of three-dimensional figures: Central axes can prime symmetry detection separately from local components. Visual Cognition, 13(3), 363-397. https://doi.org/10.1080/13506280500162490 


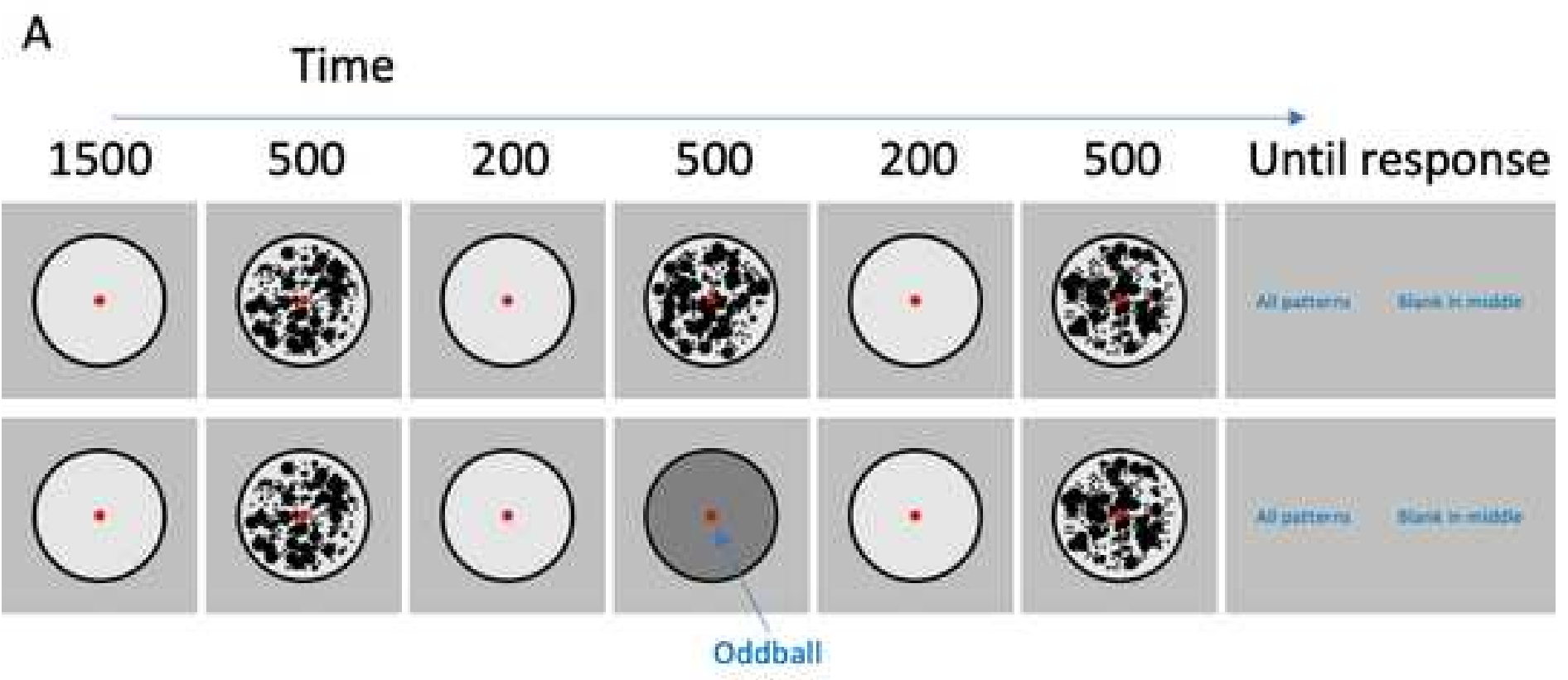

B Complex
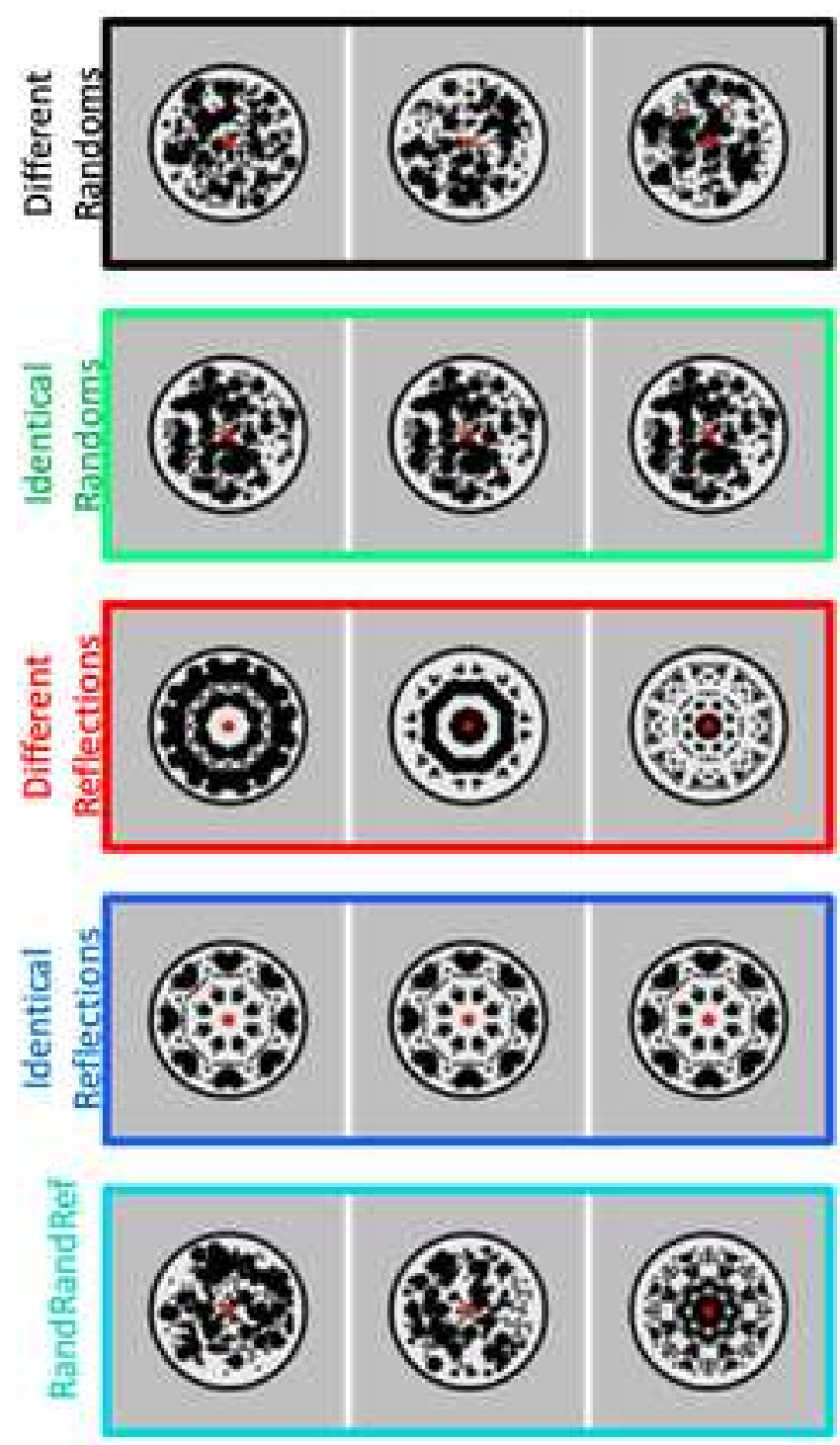

Simple
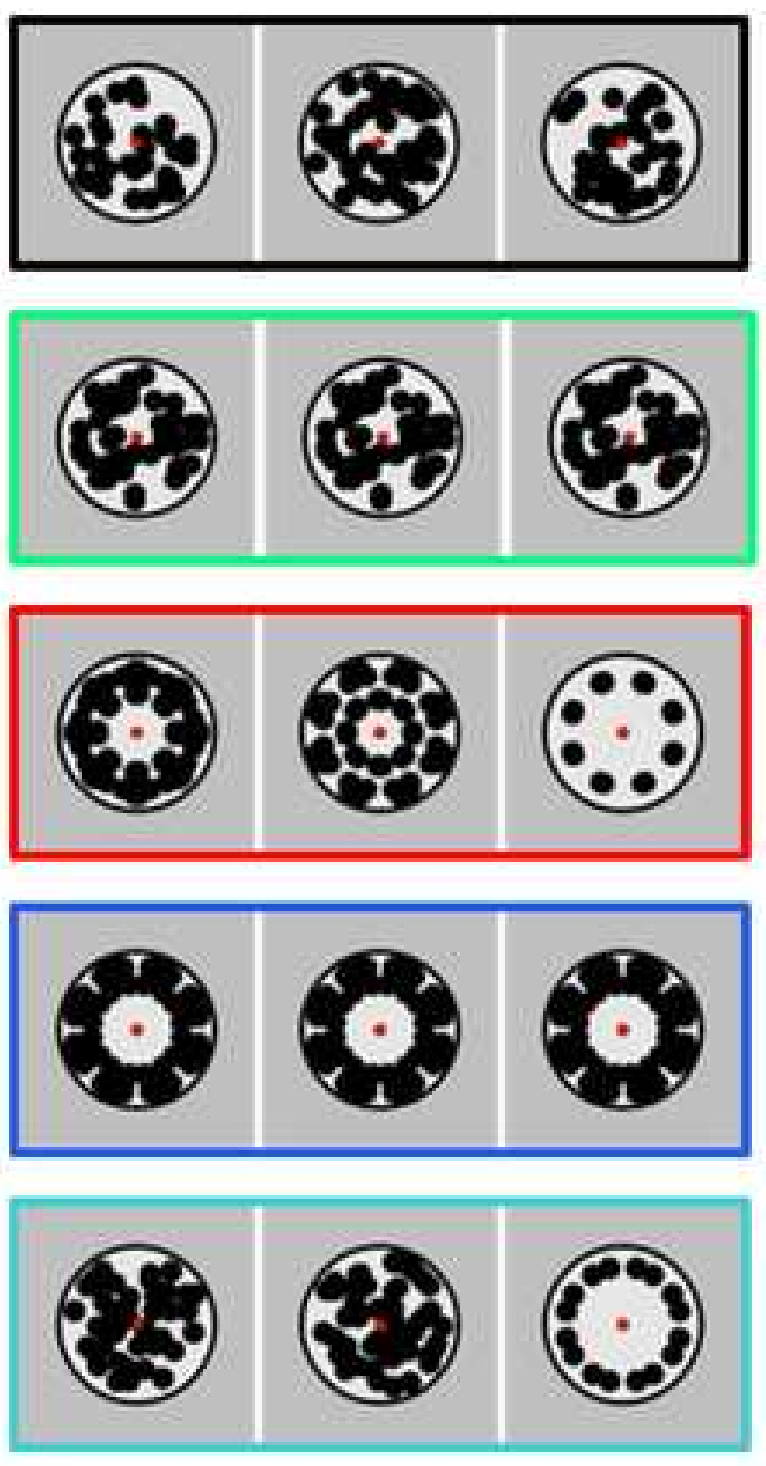

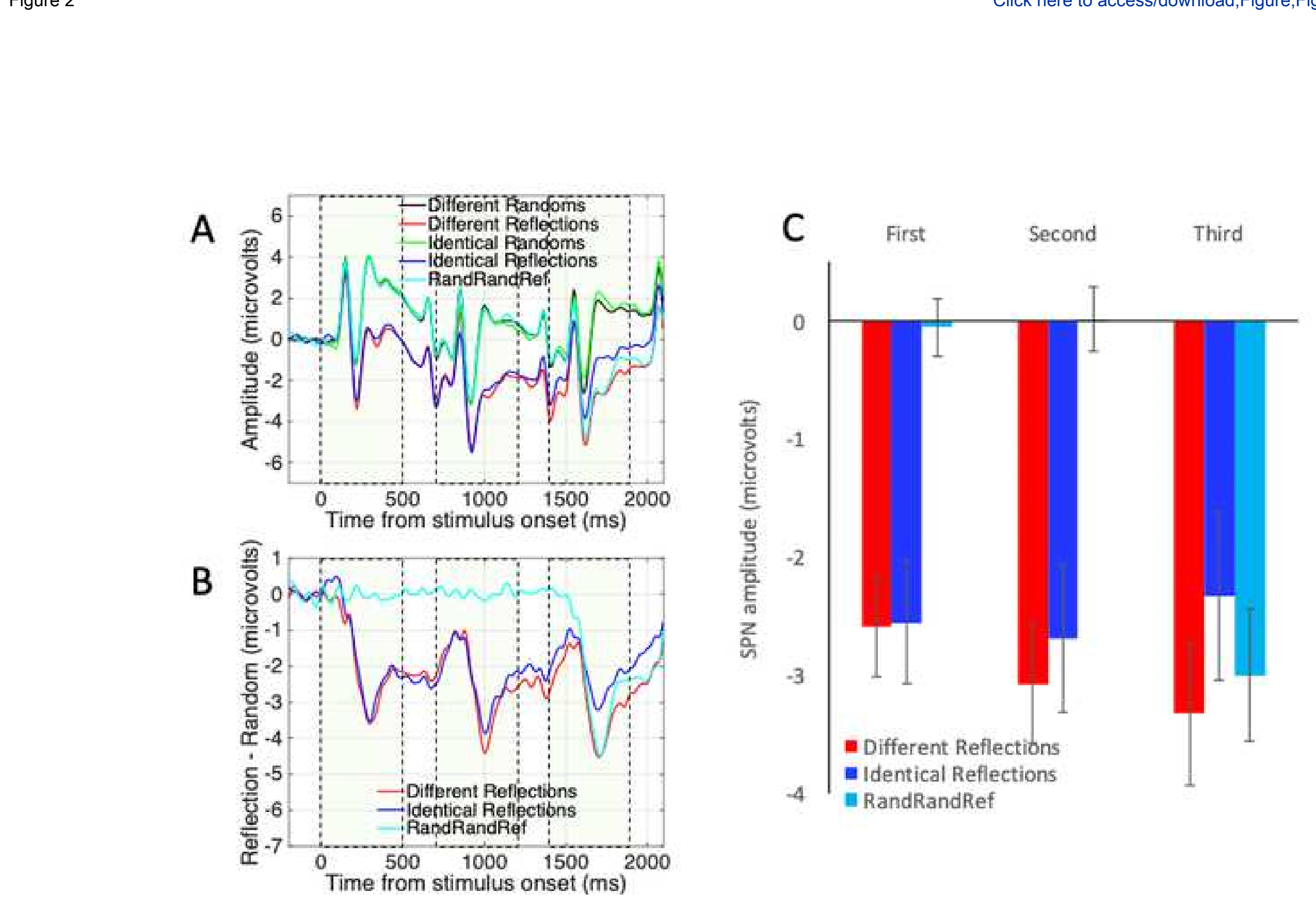


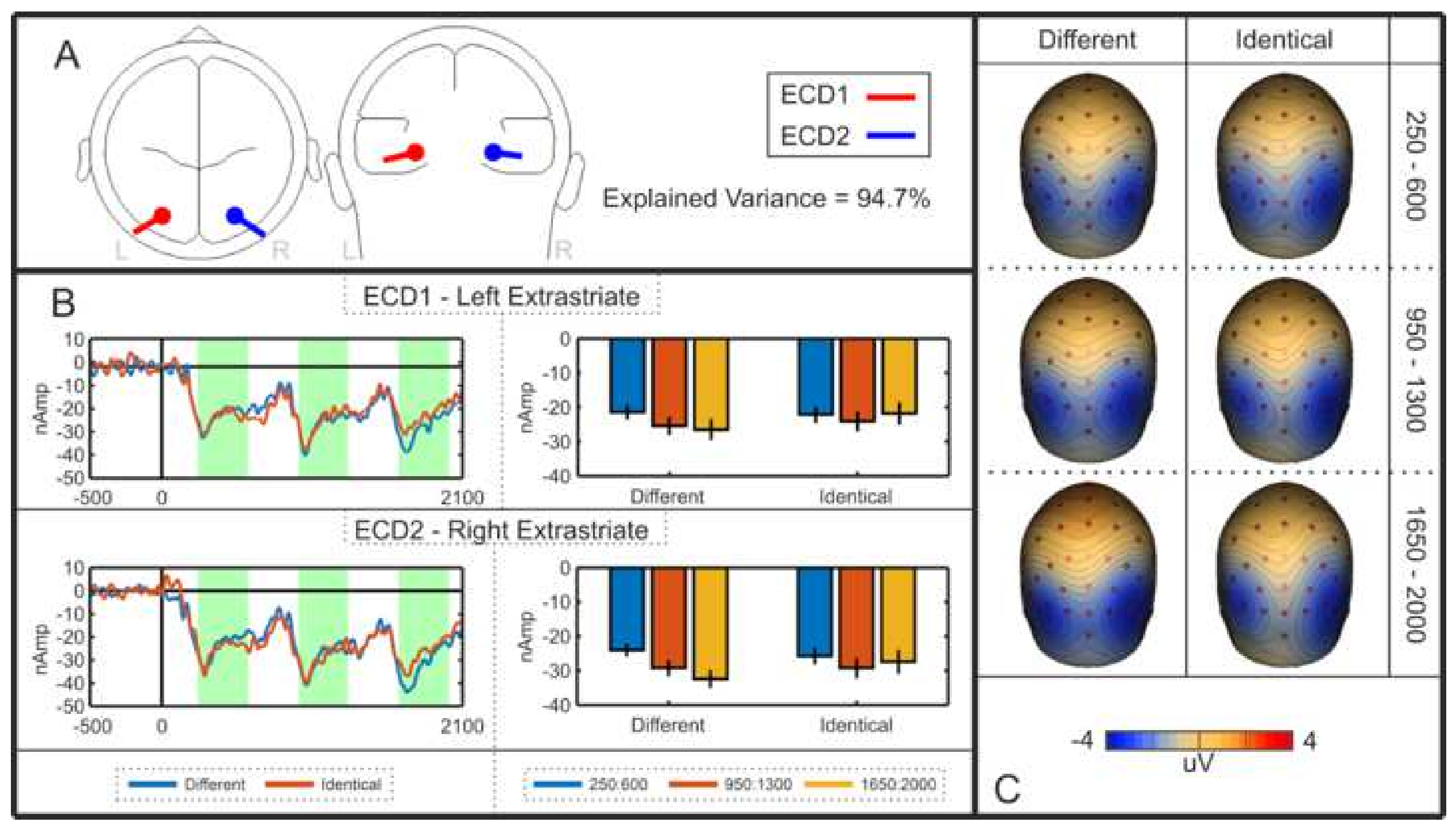



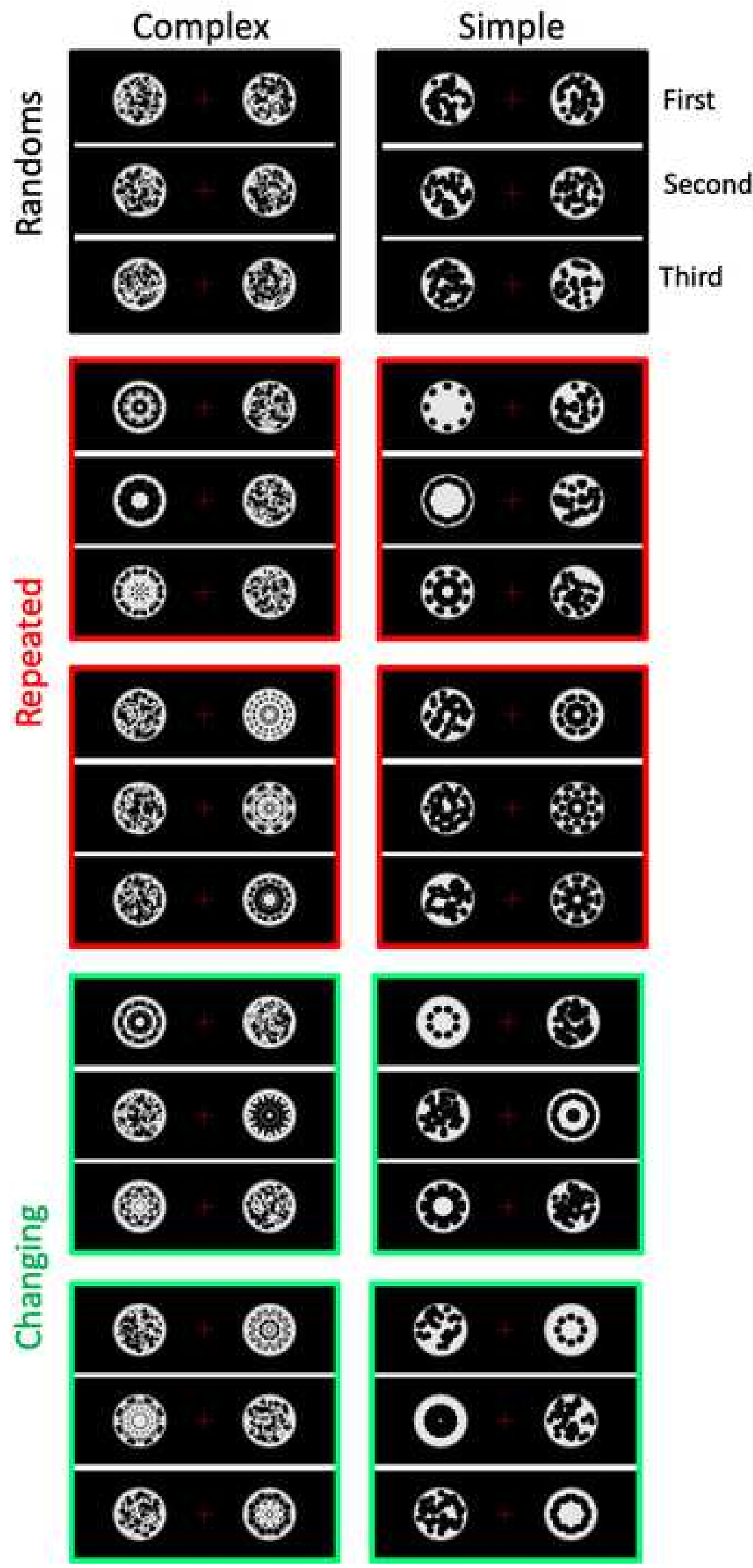

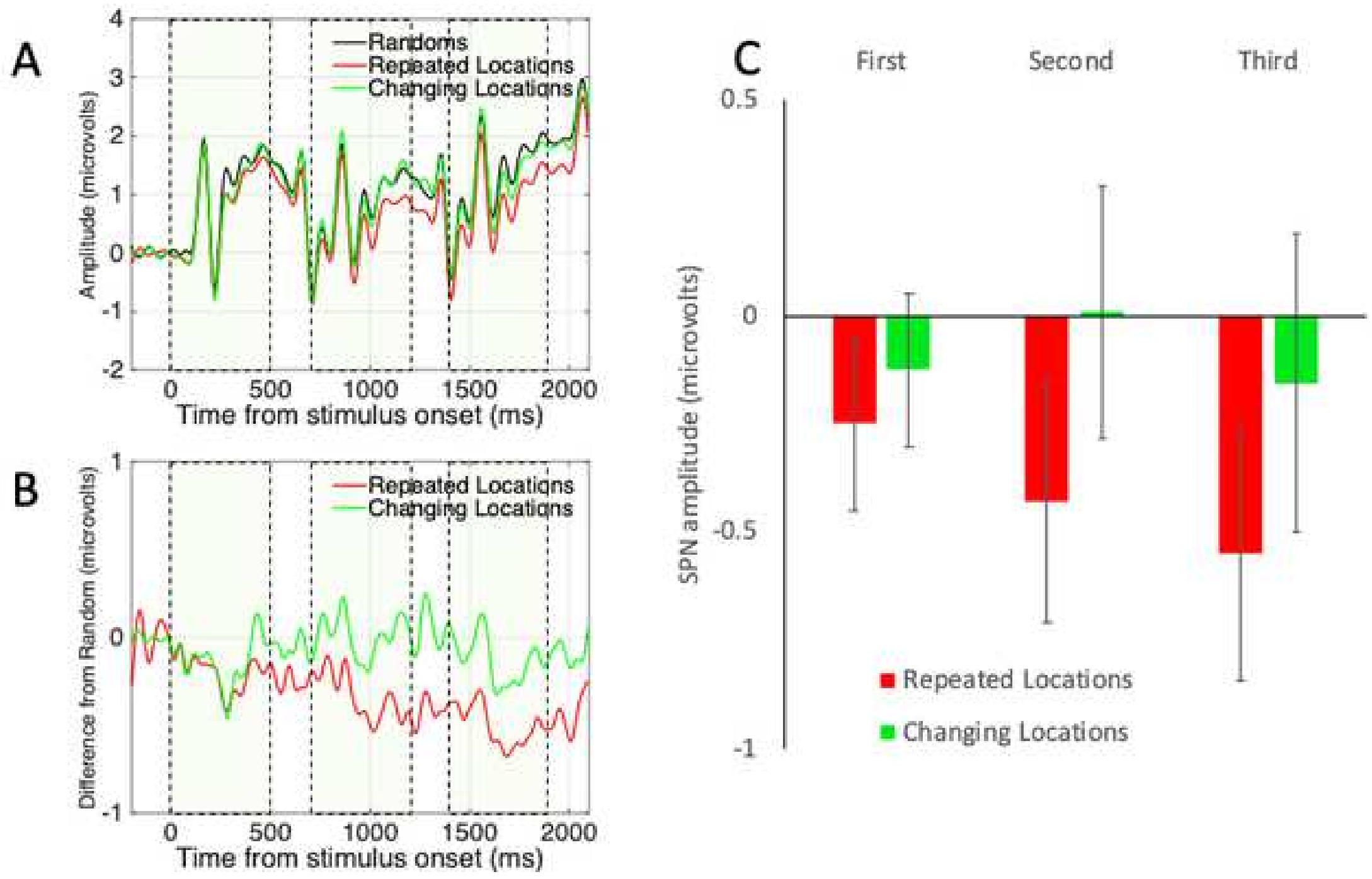

$=$ Changing Locations 


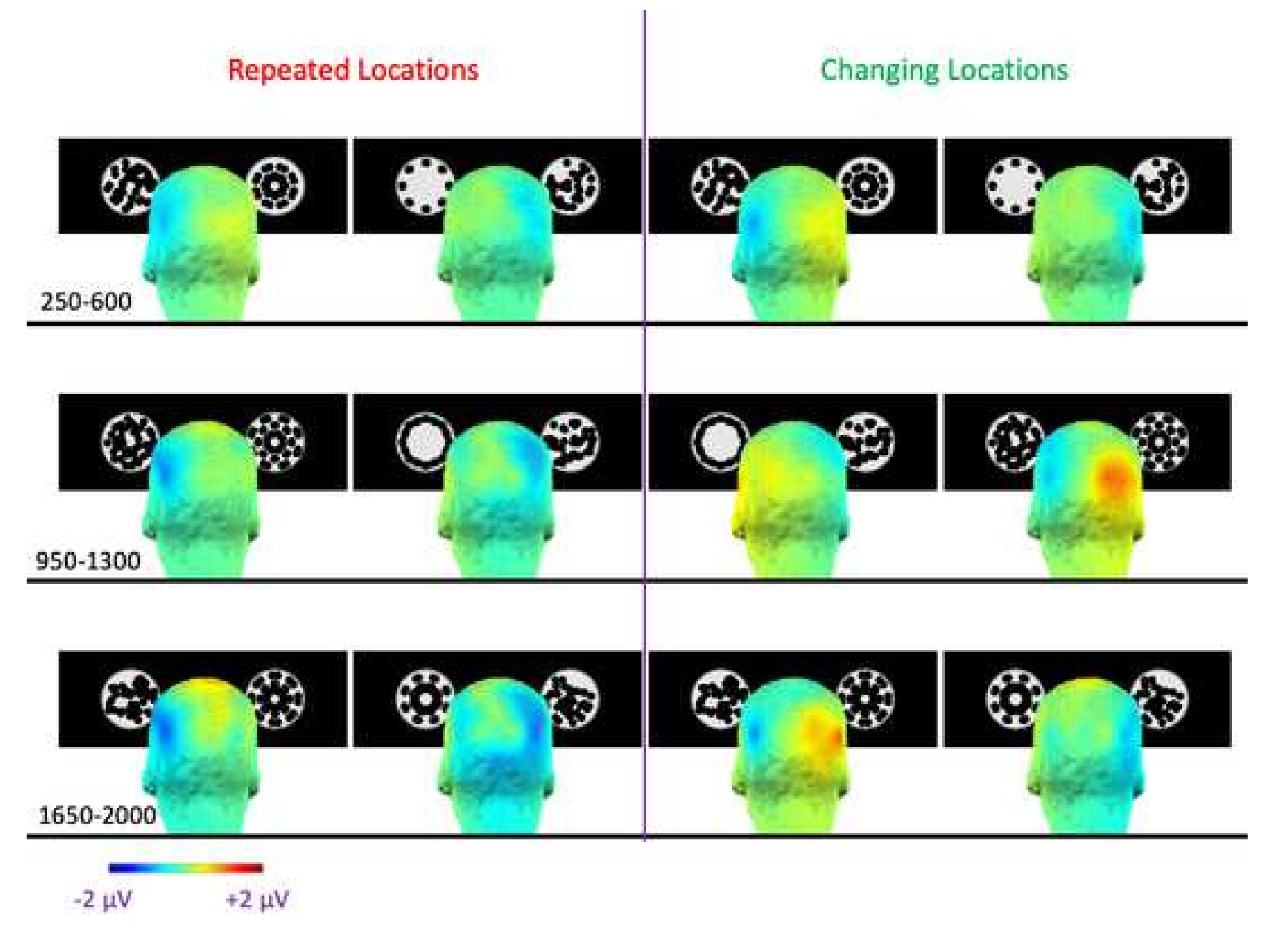




\section{Complex}
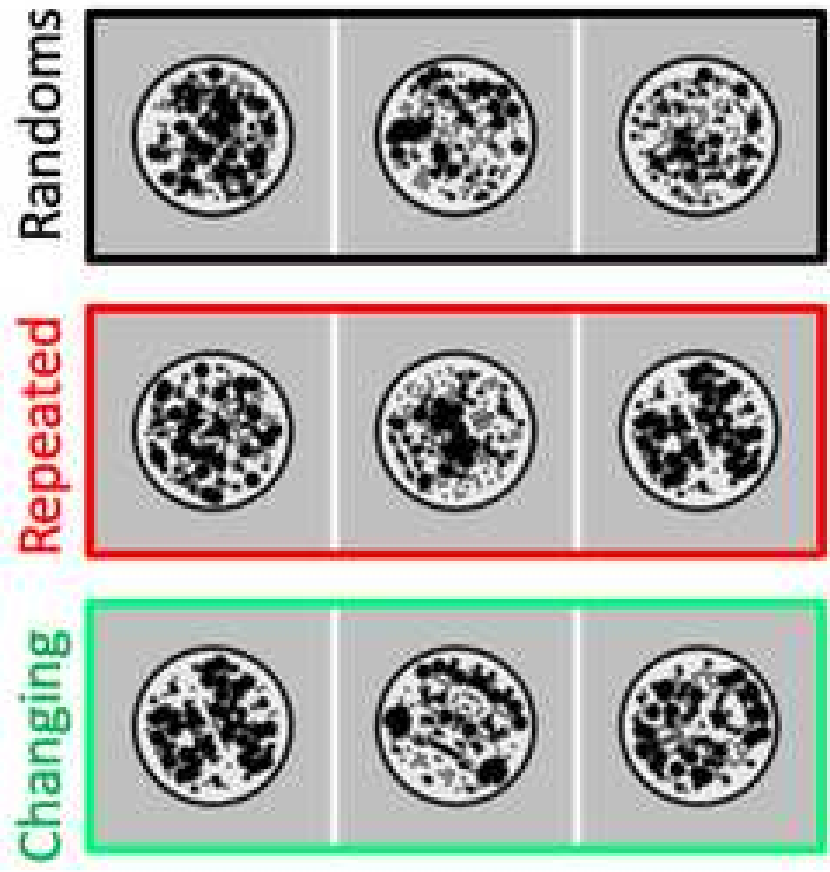

Simple
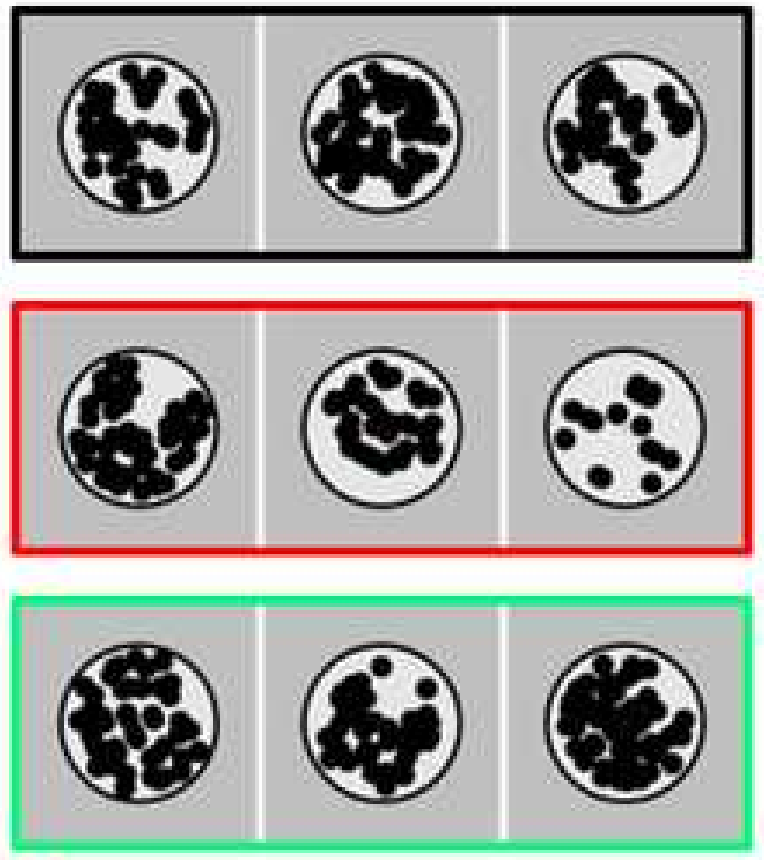

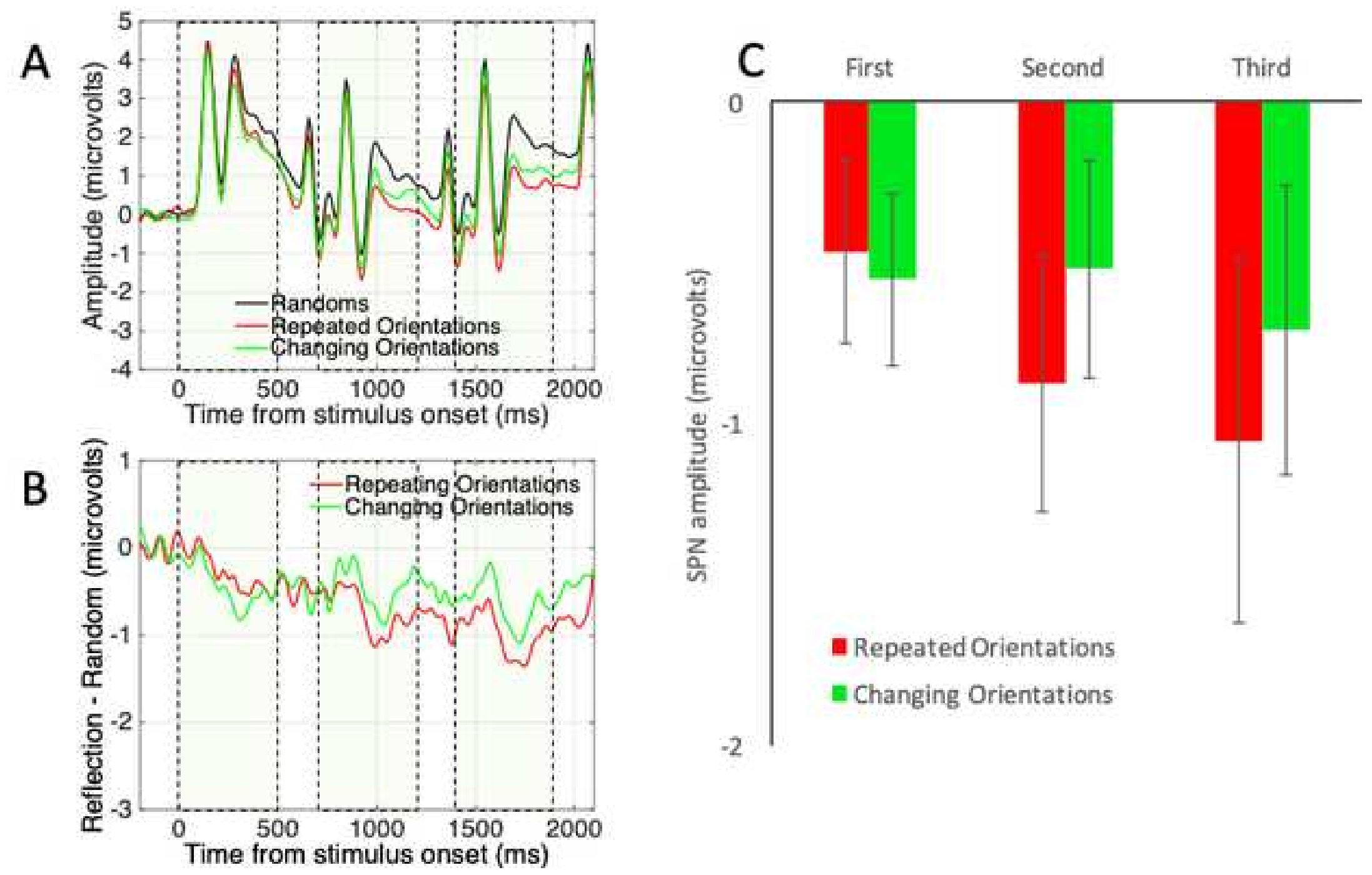
Complex
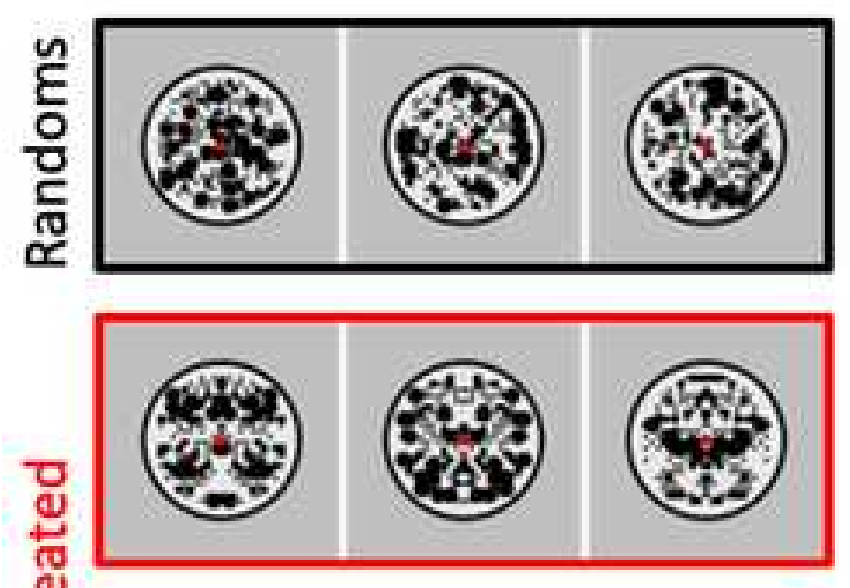

을
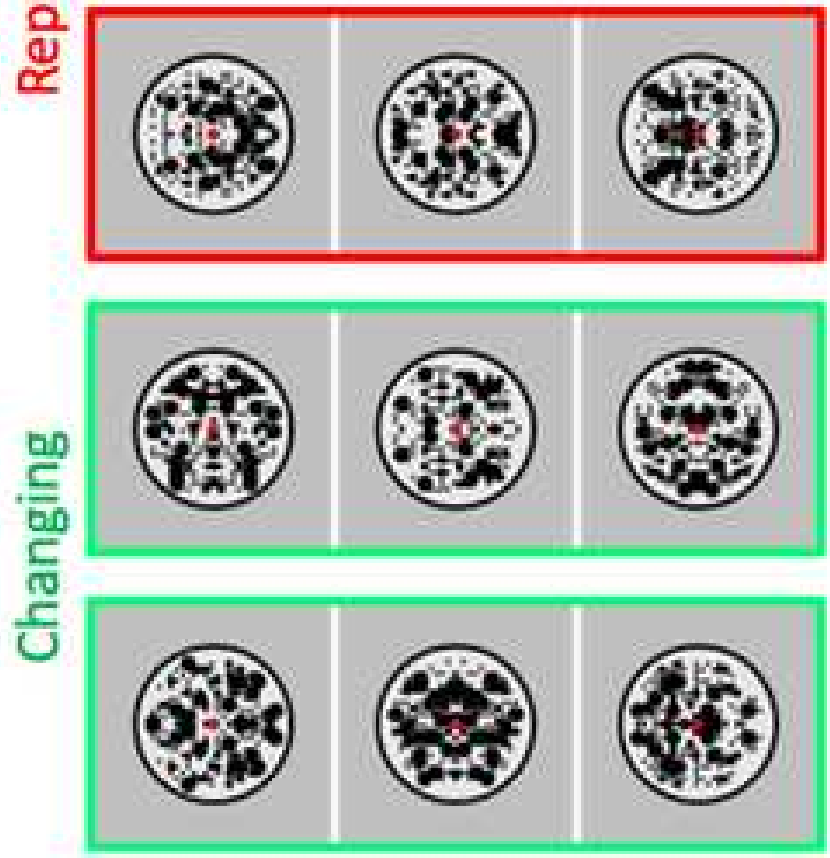

Simple
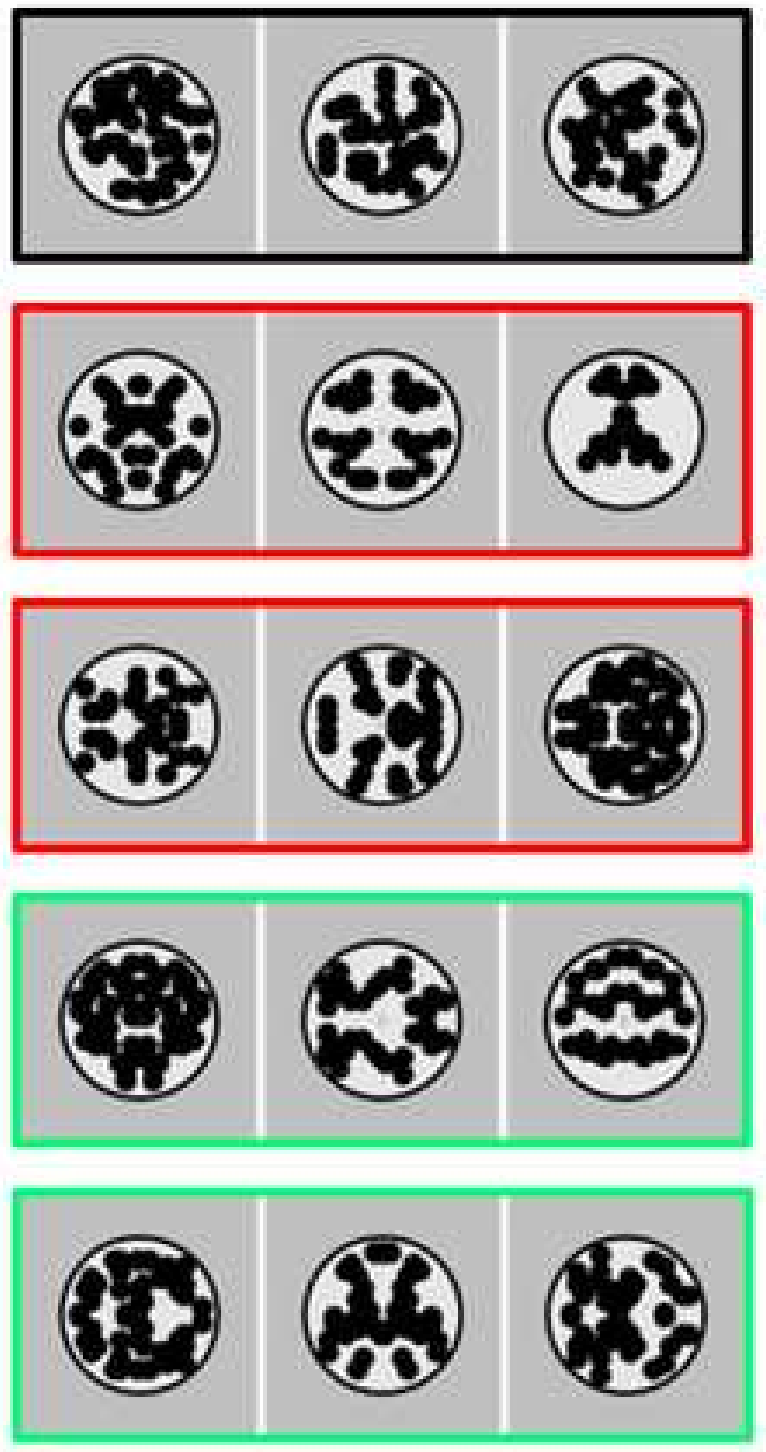

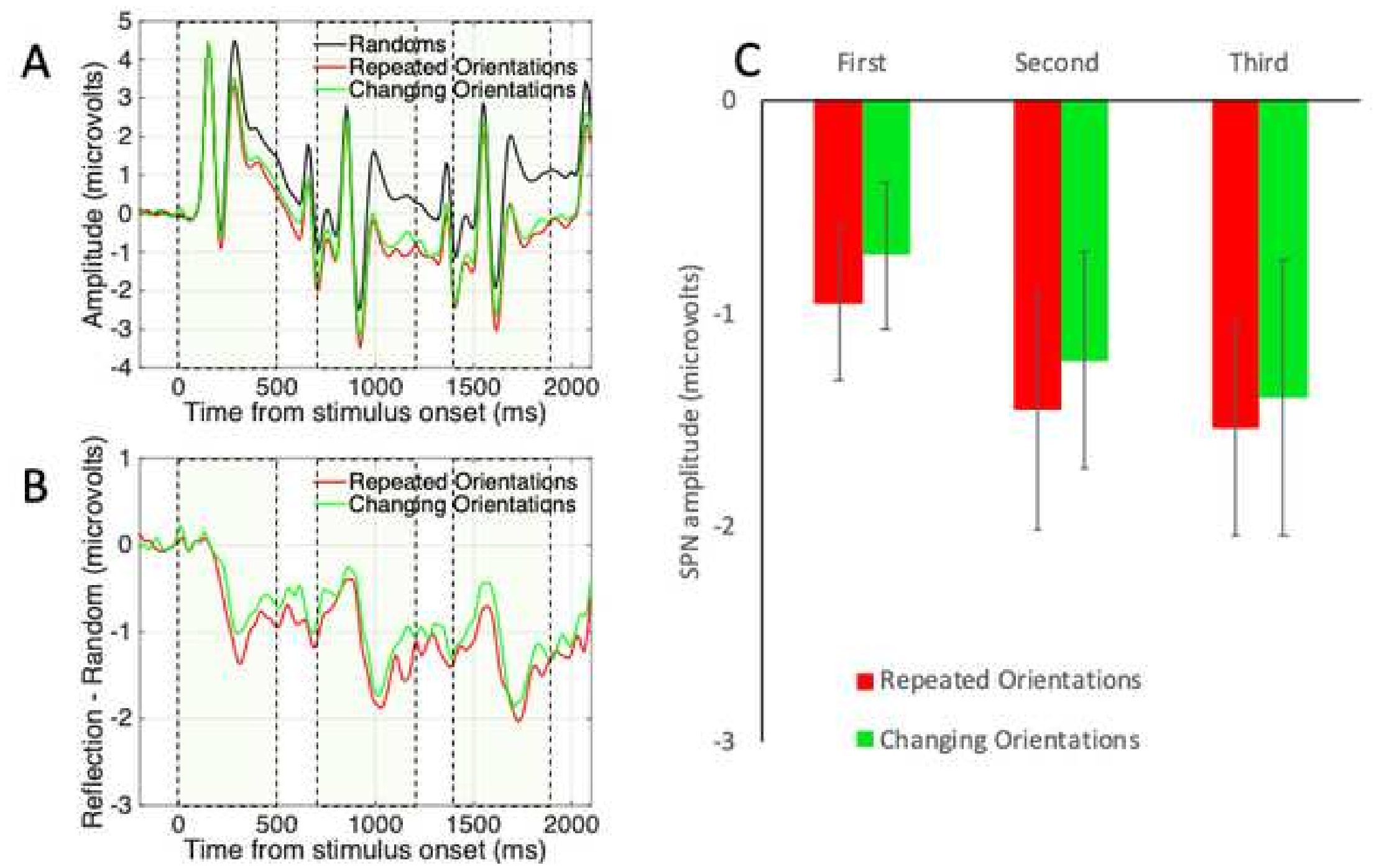

Changing Orientations 


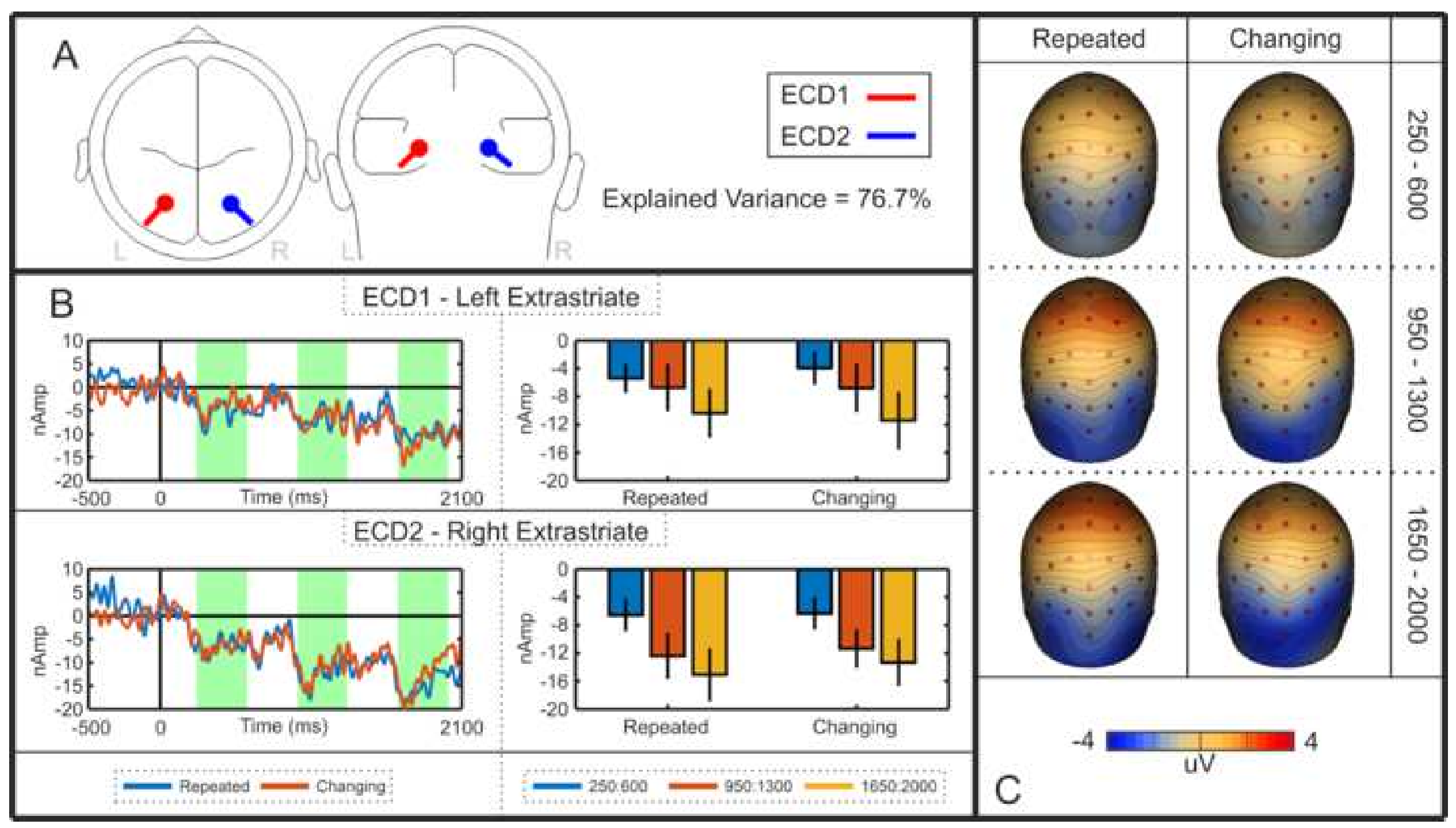


Complex
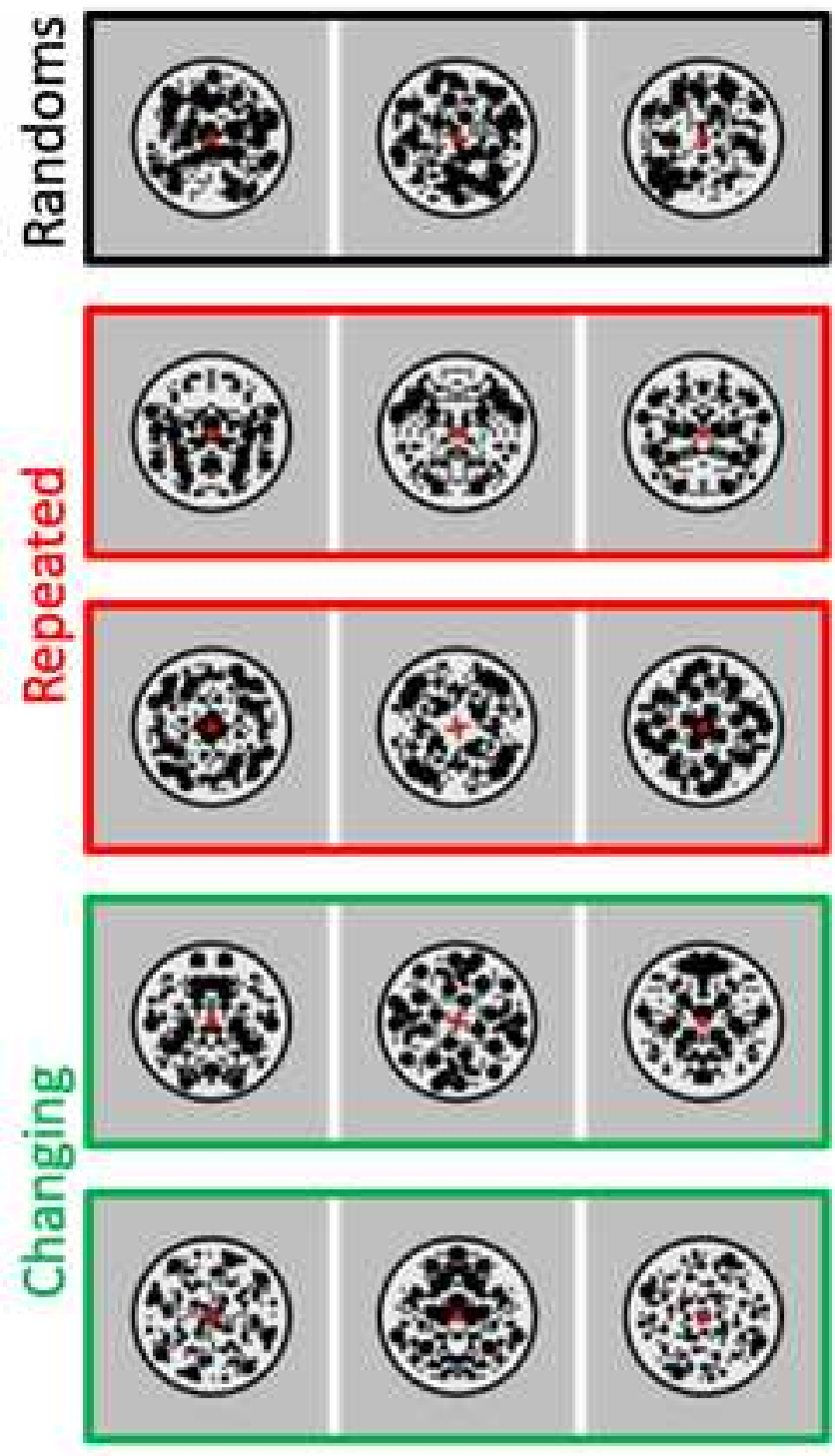

Simple
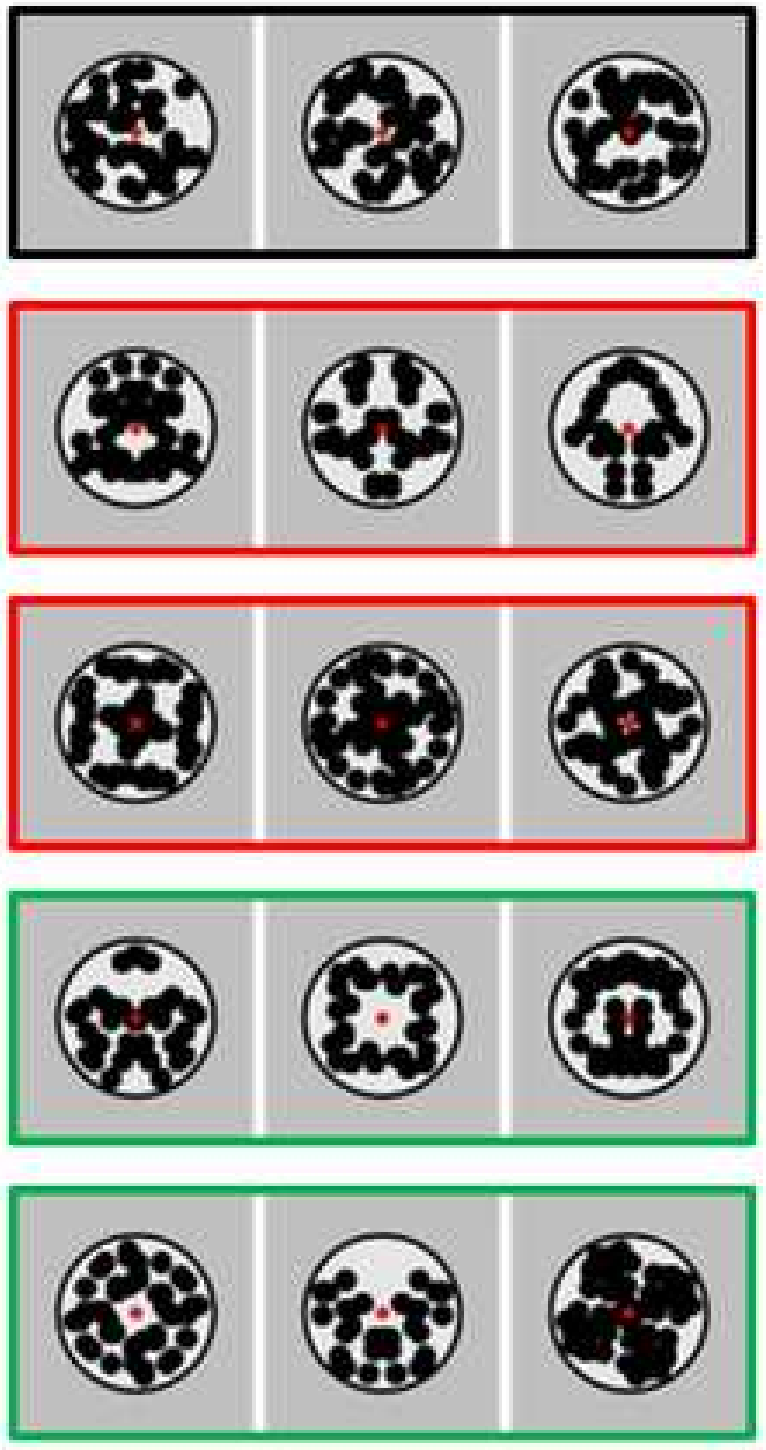

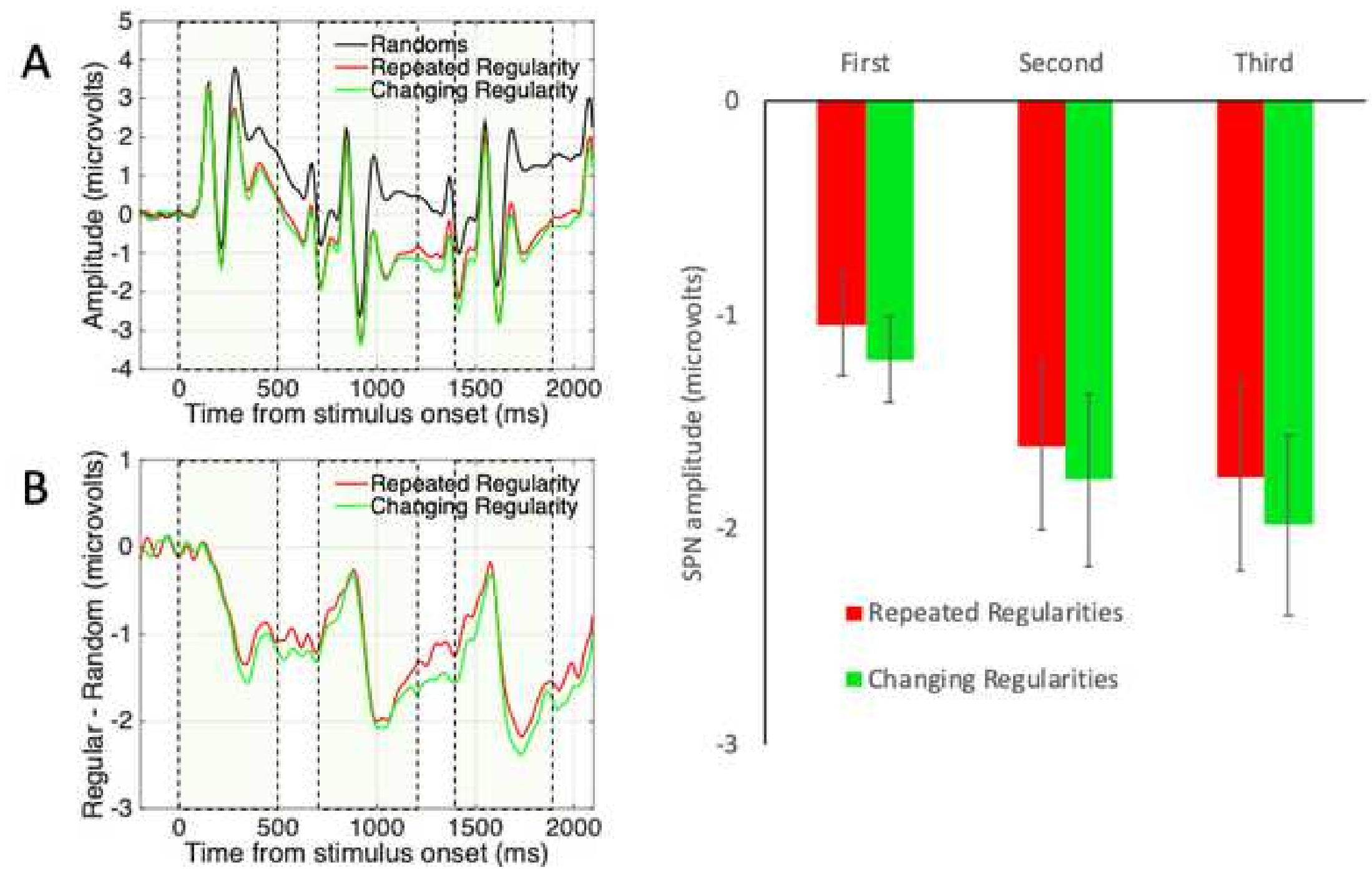


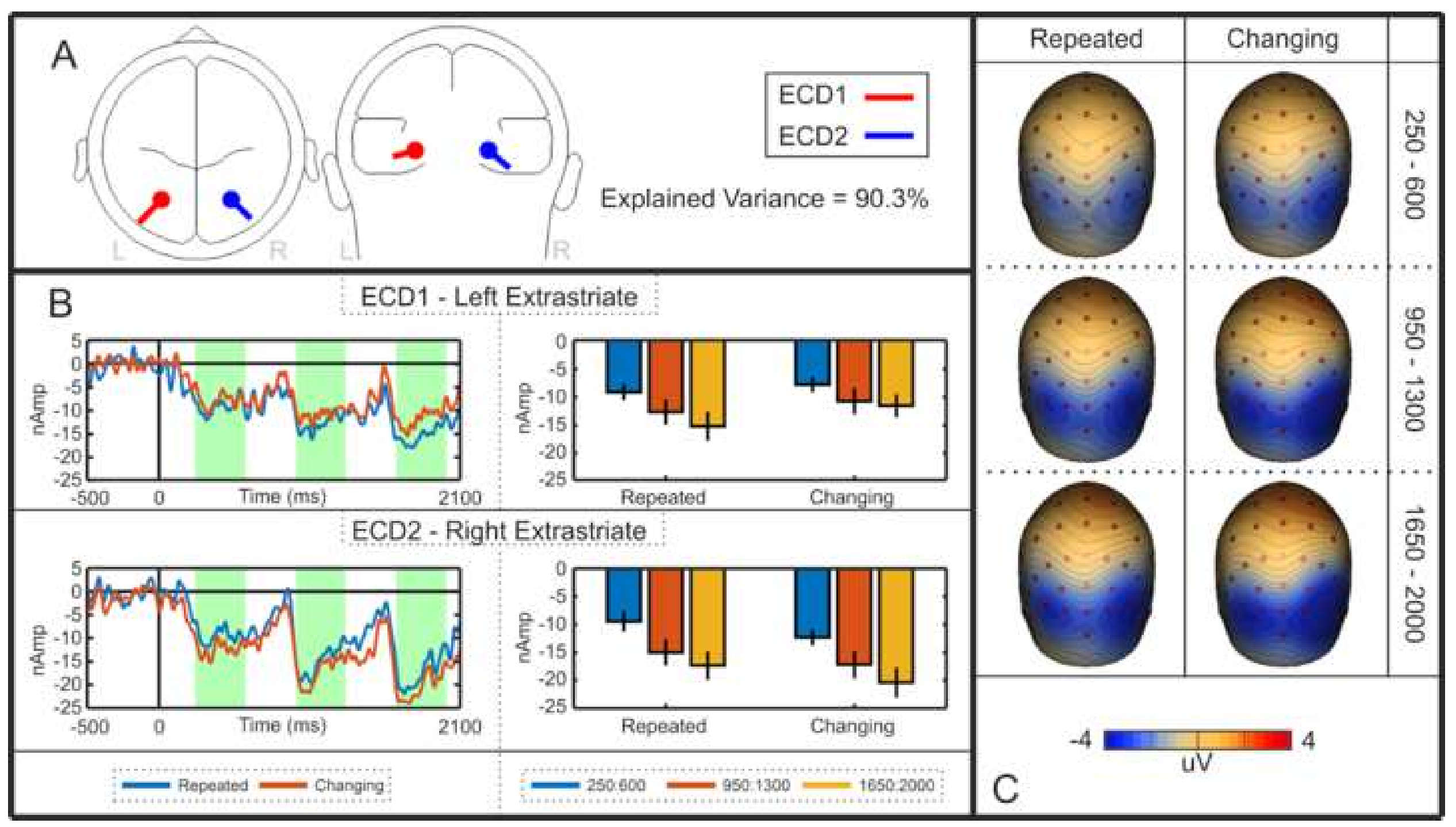


Click here to access/download Suppl. material for online publication only Supplementary materials 1. Mass Univariate.docx 
Click here to access/download Suppl. material for online publication only Supplementary materials 2 . Eye tracking analysis.docx 